\title{
Video Data Mining: Semantic Indexing and Event Detection from the Association Perspective
}

\author{
Xingquan Zhu, Member, IEEE, Xindong Wu, Senior Member, IEEE, Ahmed K. Elmagarmid, \\ Senior Member, IEEE, Zhe Feng, and Lide Wu, Senior Member, IEEE
}

\begin{abstract}
Advances in the media and entertainment industries, including streaming audio and digital TV, present new challenges for managing and accessing large audio-visual collections. Current content management systems support retrieval using low-level features, such as motion, color, and texture. However, low-level features often have little meaning for naive users, who much prefer to identify content using high-level semantics or concepts. This creates a gap between systems and their users that must be bridged for these systems to be used effectively. To this end, in this paper, we first present a knowledge-based video indexing and content management framework for domain specific videos (using basketball video as an example). We will provide a solution to explore video knowledge by mining associations from video data. The explicit definitions and evaluation measures (e.g., temporal support and confidence) for video associations are proposed by integrating the inherent feature of video data. Our approach uses video processing techniques to find visual and audio cues (e.g., court field, camera motion activities, and applause), introduces multilevel sequential association mining to explore associations among the audio and visual cues, classifies the associations by assigning each of them with a class label, and uses their appearances in the video to construct video indices. Our experimental results demonstrate the performance of the proposed approach.
\end{abstract}

Index Terms-Video mining, multimedia systems, database management, knowledge-based systems.

\section{INTRODUCTION}

$\mathrm{O}$ RGANIZATIONS with large digital assets have a need to retrieve meaningful information from their digital collections. Applications such as digital libraries, video-ondemand systems, and interactive video applications introduce new challenges in managing large collections of audiovisual content. To help users find and retrieve relevant video more effectively and to facilitate new and better ways of entertainment, advanced technologies must be developed for indexing, filtering, searching, and mining the vast amount of videos. Motivated by these demands, many video research efforts have been made on exploring more efficient content management systems. A simple framework is to partition continuous video frames into discrete physical shots and extract low-level features from video shots to support activities like searching, indexing [42], [43], or retrieval [1]. Unfortunately, a single shot which is separated from its context has less capability of conveying semantics. Moreover, the index considering only visual similarities

- X. Zhi is with the Department of Computer Science, University of Vermont, 33 Colchester Ave., Votey 377, Burlington, VT 05401. E-mail:xqzhu@cs.uvm.edu.

- $X$. Wu is with the Department of Computer Science, University of Vermont, 33 Colchester Ave., Votey 351, Burlington, VT 05401. E-mail: xwu@cs.uvm.edu.

- A. Elmagarmid is with the Department of Computer Science, Purdue University, 250 N. University Street, West Lafayette, IN 47907. E-mail:ake@purdue.edu.

- Z. Feng and L. Wu are with the Department of Computer Science, Fudan University, 220 Handan Road, Shanghai 200433, P.R. China.

E-mail: \{zhfeng, ldwu\}@fudan.edu.cn.

Manuscript received 13 Oct. 2003; revised 15 Apr. 2004; accepted 20 Oct. 2004; published online 17 Mar. 2005.

For information on obtaining reprints of this article, please send e-mail to: tkde@computer.org, and reference IEEECS Log Number TKDE-0201-1003. ignores the temporal information among shots. Consequently, the constructed cluster nodes may contain shots that have considerable variances both in semantics and visual content and, therefore, do not make much sense to human perception. The solution to this problem is to explore video knowledge to construct a database indexing structure which can facilitate database management and access. However, despite the fact that video was invented for more than 50 years ago and has been widely accepted as an excellent and popular tool to represent information, one can finds that it has never been an easy operation to extract or explore knowledge from video data [2], [3], [4], [5].

Recently, there has been a trend of employing various data mining techniques [6], [7], [8], [9], [10], [11], [12], [13], [14], [15], [16], [17], [18], [19] in exploring knowledge from large video sets. These efforts are motivated by successful data mining algorithms and by the tremendous appeal of efficient video database management. Consequently, many video mining approaches have been proposed, which can be roughly classified into three categories:

1. Special pattern detection [6], [7], [8], [9], [16], [17], [18], which detects special patterns that have been modeled in advance, and these patterns are usually characterized as video events (e.g., dialog, or presentation).

2. Video clustering and classification [10], [11], [12], [15], [19], which clusters and classifies video units into different categories. For example, in [10], [11], video clips are grouped into different topic groups, where the topic information is extracted from the transcripts of the video. 


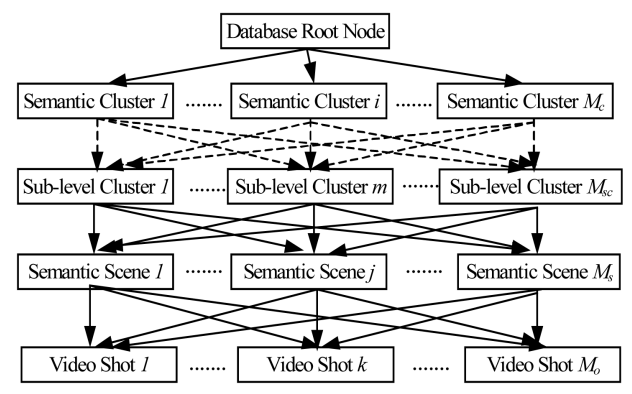

Fig. 1. The proposed hierarchical video database model.

3. Video association mining, where associations from video units are used to explore video knowledge [13], [14].

An intuitive solution for video mining is to apply existing data mining techniques [20], [21], [22] to video data directly. Nevertheless, as we can see from the three types of video mining techniques above, except [13], [14] which have integrated traditional sequential association mining techniques, most others provide their own mining algorithms. The reason is that almost all existing data mining approaches deal with various databases (like transaction data sets) in which the relationship between data items is explicitly given. Video and image databases (or other multimedia data) are different from them. The greatest distinction between video and image databases is that the relationship between any two of their items cannot be explicitly (or precisely) figured out. Although we may now retrieve video frames (and even physical shots) with satisfactory results, acquiring relationships among video frames (or shots) is still an open problem. This inherent complexity has suggested that mining knowledge from multimedia materials is even harder than from general databases [7], [23], [24], [44].

In this paper, we first introduce a knowledge-based video indexing framework to facilitate video database management and access. To explore video knowledge in supporting this framework, we propose a solution for a new research topic, video association mining, in which video processing and existing data mining algorithms are seamlessly integrated to mine video knowledge. We will systematically address the definitions and evaluation measures (temporal distance, temporal support, and confidence) for video associations by taking the distinct features of video data into consideration and by proposing a solution in mining sequential patterns from the video stream that usually consists of multiple information sources (e.g., image, audio, and caption text). We use basketball videos as our test bed because sports video generates large interest and high impact worldwide.

The paper is organized as follows: In Section 2, we present a knowledge-based video indexing framework and introduce the system architecture for video association mining. We provide several techniques in Section 3 to explore visual and audio cues that can help us bridge the semantic gap between low-level features and video content. In Section 4, we present a video association mining scheme. We discuss algorithms to classify video associations and construct video indexing in Section 5. Section 6 presents the results of our performance evaluation.

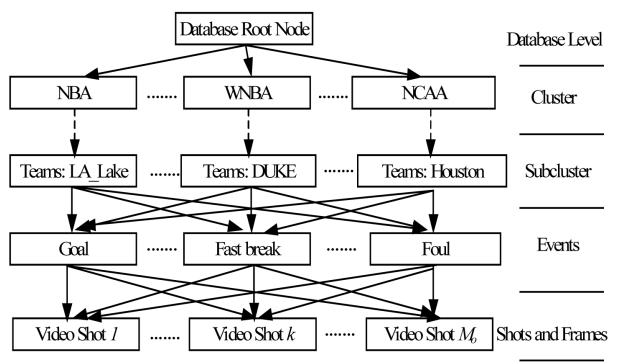

Fig. 2. Knowledge-based basketball video database management.

\section{KNOWLedge-BASEd Video INDEXING AND SYSTEM ARCHITECTURE}

There are two widely accepted approaches for accessing video in databases: shot-based and object-based. In this paper, we focus on the shot-based approach. In comparison with traditional video database systems that use low-level similarities among shots to construct indices, a semantic video database management framework has been proposed in Fig. 1, where video semantic units (scenes or story units) are used to construct database indices [7]. However, this scheme works on videos with content structure, e.g., movies and news, where video scenes are used to convey scenarios and content evolution. For many other videos, such as sports videos, there are no such story units. Instead, they contain various interesting events, e.g., a goal or a fast break, which could be taken as highlights and important semantics. Accordingly, by integrating the existing framework in Fig. 1, we propose a knowledge-based video indexing framework for basketball videos, as shown in Fig. 2. To support efficient video indexing, we need to address the following three key problems before we can actually adopt the framework in Fig. 2: 1) How many levels should be included in the model? 2) Which kinds of decision rules should be used at each node? 3) Do these nodes make sense to human beings?

We solve the first and third problems by deriving knowledge from domain experts (or from extensive observations) and from the video concept hierarchy. For basketball videos, we first classify them into a two-level hierarchy. The first level is the host association of the games, e.g., NBA, NCAA, and CBA, and the second level consists of teams of each association, such as LA_Lake and Houston, where each video can be explicitly classified into one node. Then, we integrate the inherent structure of video content to construct lower-level indices. As we have stated above, extensive observations and existing research efforts suggest that there are many interesting events in sports videos that can be used as highlights [16], [25], [26], [29]. For basketball videos, the events that likely attract most viewers' interests are goals, fast breaks, and free throws, etc. We can therefore use these events as nodes at the third level of our indexing structure. At the lowest level, we use the video shots as index nodes, as shown in Fig. 2, where each shot may have more than one parent node because some shots contain several events.

To solve the second problem, we find that the decision rules for the first two levels (cluster and subcluster) and the lowest level (shots and frames) are relatively easy and we can employ domain knowledge and some video shot segmentation algorithms [1], [27] to get satisfactory results. Our analysis in Section 3.2 also indicates that, by using the caption text in basketball videos, we can recognize team 


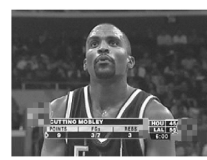

(a)

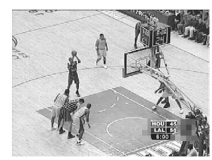

(b)

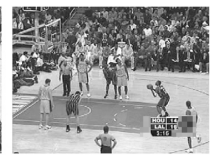

(c)

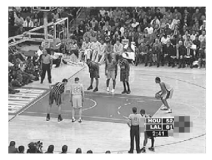

(d)
Fig. 3. Examples of the free throws of "foul shots," where shot (b) is captured right after shot (a).

names and their scores. Hence, the decision rules for the second level can also be accomplished automatically. Nevertheless, the most challenging task comes from the decision rules of the third level (events), i.e., mapping physical shots to various event nodes. In this paper, we will adopt video association mining to detect sports events. Our system architecture is given in Fig. 4, where various features are outlined below:

1. A video association mining algorithm to explore video knowledge. It also explores a new research area in video mining, where existing video processing techniques and data mining algorithms are seamlessly integrated to explore video content.

2. An association-based video event detection scheme to detect various sports events for database indexing. In comparison with other video event detection techniques, e.g., special pattern detection [25], the Hidden Markov Models [16], [29], and classification rules [28], the association-based technique does not need to define event models in advance. Instead, the association mining will help us explore models (associated patterns) from video.

3. A knowledge-based sports video management framework to support effective video access. The inherent hierarchical video classification and indexing structure can support a wide range of granularity levels. The organization of visual summaries is also inherently supported. Hence, a naive user can browse only a portion of highlights (events) to get a concise summary.

By integrating the video knowledge in the indexing structure, the constructed video database system will make more sense in supporting the retrieval and browsing for naive users. As shown in Fig. 3, where we provide four examples of "foul shots," it can be seen that the visual perception of these four shots vary a lot (especially for Fig. 3a and all others), but Fig. 3a and Fig. $3 b$ both cover the same event of the same player, which are captured from different angles. With traditional video indexing mechanisms, these four shots will be indexed at different nodes (because they have different visual perceptions) and providing Fig. 3a as the query example may never work out results, like Fig. $3 b$ (even if they do match with each other in semantics). With knowledge-based indexing, we can index them as one node (as long as we can detect this type of event), so the retrieval, browsing, and database management can be facilitated. When searching from a database constructed with the proposed indexing structure, the search engine can either include or exclude any index level to facilitate different types of queries. For example, if a user wants to query for a foul shot, regardless of the team names or the host association of the games (NBA, NCAA, etc.), the search engine can inherently attain this goal by ignoring the first two levels of indexing (cluster and subcluster in Fig. 2) at the search stage.

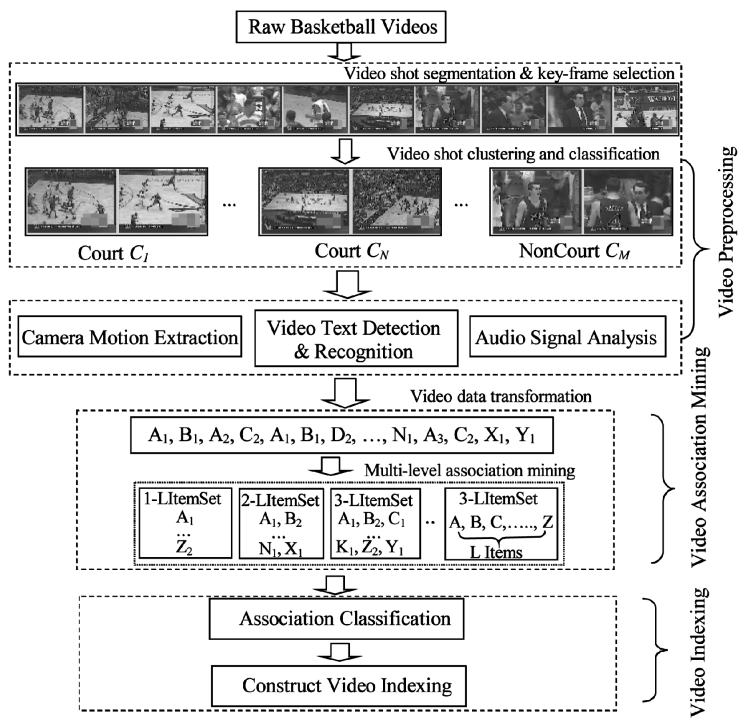

Fig. 4. The architecture of association-based video indexing.

In the system architecture in Fig. 4, we first parse a video sequence into physical shots and use a clustering algorithm to merge visually similar shots into groups. We then use dominant color detection to identify video groups that consist of court field shots and classify video shots into two categories: court and noncourt. We also perform camera motion extraction, audio signal analysis, and video text detection and recognition to detect visual and audio cues. A hybrid sequence is constructed by integrating the temporal order and the audio and visual cues of each shot. An association mining scheme is designed to mine sequential associations from the sequence. Finally, we classify all mined associations and use them to construct video indexing.

\section{Video Preprocessing}

To apply existing data mining techniques on video data, one of the most important steps is to transform video from nonrelational data into a relational data set. To facilitate this goal, we adopt a series of algorithms to explore audio and visual cues. We start with a raw video sequence and output symbolic sequences that indicate where and what types of cues appear in the video.

\subsection{Video Shot Detection and Classification}

Physical video shots that are implicitly related to content changes among frames are widely used in various video database systems [1]. To support shot-based video content access, we have developed a shot cut detection technique [27], which uses color features in each frame to characterize content changes among frames. The boundaries of shots are then determined by a threshold that is determined adaptively by using a small window (30 frames in our current work).

After shot segmentation, we try to classify each shot into two categories: court and noncourt. We first cluster visually similar shots into groups and then use the dominant color to identify groups which consist of court field shots because the court field in most sports can be described by one distinct dominant color [29]. To facilitate this goal, we use the 10th frame of each shot as its representative frame (key- 
frame $)^{1}$ and then extract two visual features from each keyframe (a 3D HSV color histogram and a 10-dimensional tamura coarseness texture [31]). When constructing a color histogram, we quantize $H, S$, and $V$ into 16,4 , and 4 bins, respectively, so that the histogram of each image is characterized by a 256-dimensional vector and the total number of feature dimensions is 266 . Given a video in the database, we assume it contains $N$ shots $S_{1}, S_{2}, \ldots, S_{N}$ and denote the key-frame of $S_{i}$ by $K_{i}$. Suppose $H_{i, l}, l \in[0,255]$, and $T C_{i, n}, n \in[0,9]$ are the normalized color histogram and texture of $K_{i}$. The distance between shots $S_{i}$ and $S_{j}$ is defined by (1), where $W_{C}$ and $W_{T}$ indicate the weight of each feature:

$$
\begin{aligned}
\operatorname{Dis}\left(S_{i}, S_{j}\right)= & W_{c}\left\{1-\sum_{l=0}^{255} \min \left(H_{i, l}, H_{j, l}\right)\right\} \\
& +W_{T} \sqrt{\sum_{n=0}^{9}\left(T C_{i, n}-T C_{j, n}\right)^{2} .}
\end{aligned}
$$

We want to group shots that are similar into a cluster. In addition, different clusters should have sufficiently different characteristics. Hence, we adopt a modified split-and-merge clustering algorithm [32] by sequentially executing two major procedures: merging and splitting. In the merging procedure, we iteratively merge the most similar clusters (defined by (2)) until the distance between the most similar clusters is larger than a given threshold. Nevertheless, this merging procedure may generate clusters with a large intracluster distance (defined by (3)). Accordingly, after the merging procedure, we turn to the splitting procedure to split clusters with large visual variances. We iteratively calculate the intracluster distance for any cluster $C_{i}$, the cluster with its intracluster distance larger than a given threshold is separated into two clusters until all clusters have their intracluster distance less than the given threshold.

Let's denote the $i$ th cluster by $C_{i}$ and the number of members in $C_{i}$ by $N_{i}$, where each element $\left(S_{i}^{l}, l=1, \ldots, N_{i}\right)$ in the cluster is a shot. The intercluster distance between $C_{i}$ and $C_{j}$ is defined by (2):

$$
d_{\min }\left(C_{i}, C_{j}\right)=\min _{S_{i}^{l} \in C_{i}, S_{j}^{k} \in C_{j} ; l=1, \ldots, N_{i}, k=1, \ldots, N_{j}} \operatorname{Dis}\left(S_{i}^{l}, S_{j}^{k}\right) .
$$

We then define the intracluster distance of $C_{i}$ by (3):

$$
d\left(C_{i}\right)=\max _{S_{i}^{l} \in C_{i}, S_{i}^{k} \in C_{i} ; l \neq k ; l=1, \ldots, N_{i}, k=1, \ldots, N_{i}} \operatorname{Dis}\left(S_{i}^{l}, S_{i}^{k}\right) .
$$

After we have clustered visually distinct shots into groups, we can use the dominant color (usually, a tone of yellow) to identify groups that consist of court field shots. However, even though the color of the court field is likely a tone of yellow, the actual color may vary from stadium to stadium and also change with lighting conditions. Therefore, we cannot assume any specific value for this dominant color, but learn it adaptively. We randomly sample $N$ frames from video sequences (in our system, we set $N=50$ ). Because sports videos usually focus on the court

1. For the sake of simplicity, we use this simplest key-frame selection mechanism. One can also adopt other complicated approaches [30] Nevertheless, because our purpose is not to characterize the content change in the video shots, but to classify video shots into different categories, we find the performance of this simple mechanism works reasonably well.

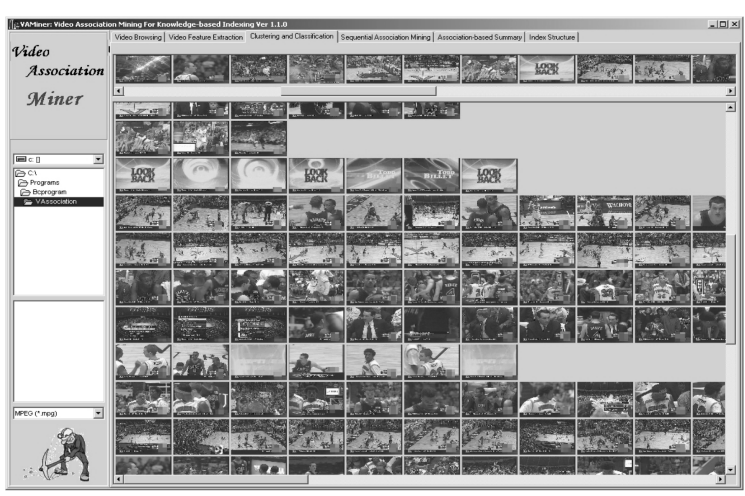

Fig. 5. Video shot clustering results where each icon image represents one shot (the first row represents the first shot of each group and all other rows represent each clustered group)

field, most of these $N$ frames will contain the court field. We then calculate the histogram of the hue component of each frame (in HSV color space). The histogram of the hue component is added up over these $N$ frames. We pick up the peak of this cumulated hue histogram and use the corresponding hue value as the court field hue color. Assuming this hue color is denoted by $\bar{H}$, we calculate the average saturation and intensity value of the pixels in these $N$ frames, where the hue color of the pixels is $\bar{H}$. We denote the average saturation and intensity by $\bar{S}$ and $\bar{I}$. For each group $G_{i}$ (acquired from the former clustering algorithm), we calculate the dominant hue color of all key-frames in $G_{i}$, denote it by $\bar{H}_{i}$, and the average saturation and intensity of the pixels with their hue color equal to $\bar{H}_{i}$ are denoted by $\bar{S}_{i}$ and $\bar{I}_{i}$. Then, we use (4) to calculate the distance between $G_{i}$ and the template. After we get the distances from all video groups, we use a simple thresholding method to classify each group into two exclusive categories: a group consisting of court filed shots or not:

$$
H \operatorname{svDis}(i)=\sqrt{\left(\bar{I}_{i}-\bar{I}\right)^{2}+\left(\bar{S}_{i}\right)^{2}+(\bar{S})^{2}-2 \bar{S}_{i} \cdot \bar{S} \cdot \cos (\theta)},
$$

$$
\theta= \begin{cases}\left|\bar{H}_{i}-\bar{H}\right| & \text { if }\left|\bar{H}_{i}-\bar{H}\right|<180^{\circ} \\ 360^{\circ}-\left|\bar{H}_{i}-\bar{H}\right| & \text { if }\left|\bar{H}_{i}-\bar{H}\right|>180^{\circ}\end{cases}
$$

Generally, since one sports video is captured from one place, both shot clustering and classification can acquire relatively good performances. As shown in Fig. 5, we can find that the shots containing the court are successfully clustered into groups (and likely characterized by cameras with different angles or views) because the court field color plays an important role in similarity evaluation.

\subsection{Video Text Detection and Recognition}

There are two types of video text: the first is the text shown in video scenes, referred to as scene text hereafter, and the second is the text postprocessed and added into the video, such as team names and their scores, which we call caption text. For sports videos, caption text is much more important than scene text because the former directly conveys video semantics. With caption text, we can acquire the name of each team and use it to construct the second level index in Fig. 2. Moreover, as long as we can detect the team scores, the score change is directly associated to the "goal" events.

In comparison with scene text, the caption text has one distinct feature: It rarely moves. This distinct feature 


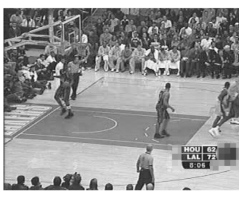

(a)

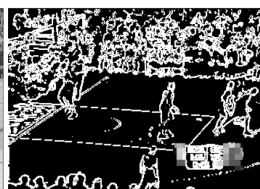

(b)

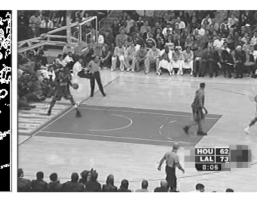

(c)

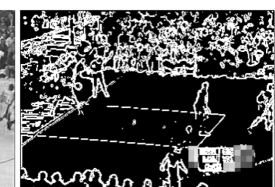

(d)

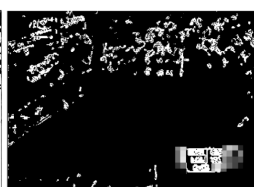

(e)

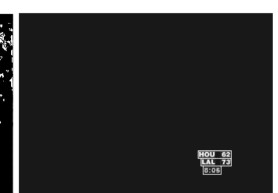

(f)

Fig. 6. Video caption text region detection, (a) frame $F_{i}$, (b) edge frame $E_{i}$, (c) frame $F_{i+\tau}$, (d), edge frame $E_{i+\tau}$, (e) the edge pixels which appear in both $E_{i}$ and $E_{i+\tau}$, and (f) detected caption text regions.

inspires us to develop a simple but efficient caption text detection algorithm:

1. Calculate the edge of the current frame $F_{i}$, denote the edge frame by $E_{i}$, and then calculate the edge of the succeeding frame with a step $\tau$ (in our system, we set $\tau=10)$, i.e, frame $F_{i+\tau}$ and its edge frame $E_{i+\tau}$.

2. Compare edge pixels in $E_{i}$ and $E_{i+\tau}$. If the edge pixel in $E_{i}$ is still the edge pixel in $E_{i+\tau}$, the current pixel is a candidate of caption text pixel.

3. After all edge pixels in $E_{i}$ have been processed, use a median filter to eliminate noise and all remaining pixels to form the caption text regions.

If the camera motion is still, we take the locations of the text regions detected from the most recent moving frame as the caption text regions in the current frame because, if the camera is still, all edge pixels in $E_{i}$ and $E_{i+\tau}$ are the same and the proposed method may not work. Meanwhile, we add another constraint: The detected caption text region should appear in either the top $\frac{1}{4}$ or bottom $\frac{1}{4}$ of the frame region. We have observed various basketball videos from ESPN, FOX, etc., and found that, in almost all situations, the team names and their scores appear in the top or the bottom regions of the frame because it has less impact with the viewers.

After candidate text regions have been detected, we need to prune out some false candidates and handle the scale problem. The regions with their height and width less than given thresholds are eliminated and the horizontal-vertical ratio of the regions should also be in a certain range. After that, we use the Bilinear Interpolation algorithm to resize each candidate region into a certain size of box and transform the pixels into binary values (black or white) for recognition.

To recognize caption text, we adopt an existing OCR (Optical Character Recognition) engine, WOCAR [33], which takes a binarized image as the input and yields an ASCII string result. This engine has many function calls to support applications. More details about video text detection can be found in [34]. Fig. 6 gives an example of our caption text detection results. Meanwhile, since we only detect team names and score numbers, we can develop a small vocabulary for the OCR engine to improve the recognition accuracy. We perform the algorithm on every $\tau(\tau=10)$ frames and use detected team names to construct the second level index. Once we detect a score change, we add a symbolic tag at the corresponding place.

\subsection{Camera Motion Characterization}

Given a shot $S_{i}$, the camera motions in the shot can also imply some knowledge. For example, a fast break usually happens when the camera is still, or pans slowly, then suddenly speeds up and pans quickly. Hence, we can explore semantic cues from camera motions in each shot.
However, the camera motions in noncourt field shots have less knowledge or can even be meaningless. We therefore only analyze camera motions from court field shots.

To extract camera motions, we have recently developed a qualitative camera motion extraction method [35]. This method works on compressed MPEG streams and uses motion vectors from $P$-frames to characterize camera motions. For any two motion vectors in each $P$-frame, we first classify their mutual relationship into four categories: approaching, parallel, diverging, and rotation, as shown in Fig. 7. Generally, if the camera pans or tilts, the mutual relationship between any two motion vectors is likely parallel, as shown in Fig. 9 and, if the camera zooms, the mutual relationship is likely to be approaching or diverging (depending on whether the actual motion is zoom-in or zoom-out). We then construct a 14-bin motion feature vector to characterize the camera motion in each $P$-frame. More details related to the camera motion classification can be found in [35]. Only certain types of camera motions in basketball videos could possibly imply useful information and we therefore classify the camera motion of each $P$-frame into the following six categories: Still, Pan (left and right), Zoom (in and out), and others. A motion description hierarchy is given in Fig. 8.

In addition to classifying the camera motion, we also calculate the average motion magnitude of each $P$-frame by (6), where $M V_{i}$ is the number of valid motion vectors in the $P$-frame $i, x_{i}(m)$ and $y_{i}(m)$ are the $x$ and $y$ components of the motion vector $m$ in the frame $i$. Our objective is to characterize the speed of motion activities. We roughly classify the motion magnitude into three categories: slow, medium, and fast, by specifying a numeric range for each category. Finally, a temporal filter is adopted to eliminate falsely detected camera motions. For the MPEG videos used in our test bed, there are eight $P$-frames in each second of stream. So, we use the dominant motion of these eight $P$-frames and its magnitude as the camera motion in this range and collect camera motions and magnitude (in the original temporal order) to form a symbolic sequence. For MPEG videos encoded with fewer $P$-frames, one can use a longer time span for temporal filtering, because the

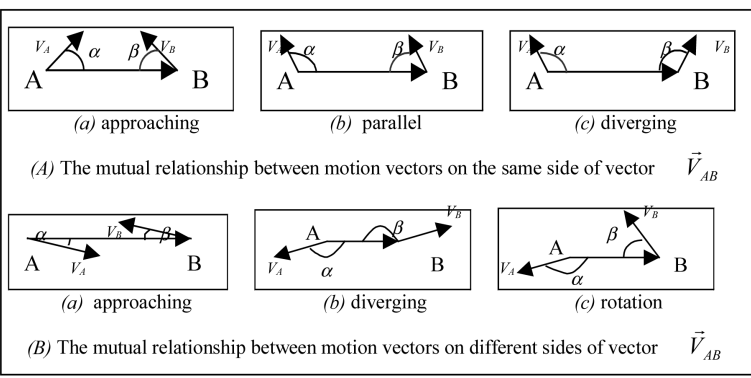

Fig. 7. Mutual relationships between two motion vector in each P-frame. 


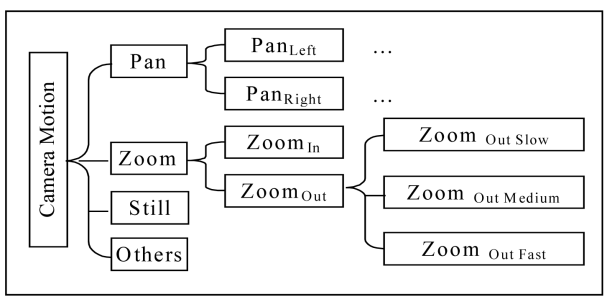

Fig. 8. Camera motion description hierarchy.

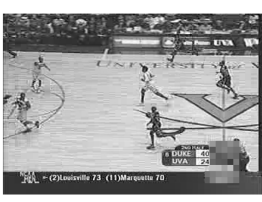

(a)

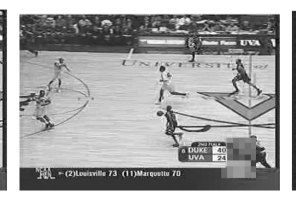

(b)

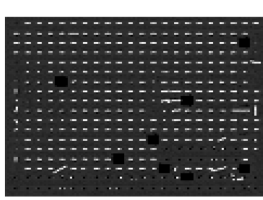

(c)
Fig. 9. (a) Camera pan operation and (b) and (c) the corresponding motion vectors.

dominant camera motion in sports video usually lasts several seconds. With the proposed approach, we can identify three typical camera motions: Pan, Tilt, and Zoom. All other camera motions are marked as "Others." For mining purposes, after camera motion detection all "Others" tags will be removed from the sequence:

$$
M(i)=\sum_{m=1}^{M V_{i}} \sqrt{x_{i}(m)^{2}+y_{i}(m)^{2}} / M V_{i} .
$$

\subsection{Salient Audio Event Detection}

In sports videos, some special audio events, e.g., audience applause and a referee's whistle, will help us acquire some semantic cues. Generally, audience applause occurs when exciting events happen, e.g., shooting and/or a goal, and a referee's whistle may imply an interruption or another special event.

To detect audience cheering, we use the pitch of audio signal. Basically, pitch is the fundamental frequency that reveals harmonic properties of audio and is an important parameter in the analysis and synthesis of speech signals. In comparison with voice and music, the pitch value of audience applause is very small. In most cases, this value in sports videos is zero because, when cheering happens, the audio signal exhibits a constant high value noise that likely drowns out other audio signals, e.g., the voice of the anchorperson or the music. We therefore extract the pitch for each audio frame. In our system, the audio frame length is $20 \mathrm{~ms}$ and the frame shift is $0 \mathrm{~ms}$. Because the duration of cheering usually exceeds 1 second, we apply cheering detection on each 1-second segment. For each segment, we calculate the NonZero Pitch Ratio (NZPR), which is defined as the ratio between the number of frames whose pitch is not zero and the total number of frames in a segment. For a cheering segment, its NZPR value likely exhibits a small value, and a simple threshold scheme can distinguish cheering segments from others. Fig. 10 shows the results of NZPR values from a test sports video with one minute duration, where four cheering events appear at 3s-9s, 20s$25 \mathrm{~s}, 41 \mathrm{~s}-44 \mathrm{~s}$, and $54 \mathrm{~s}-57 \mathrm{~s}$.

To detect a referee's whistle, we use spectrum domain features. Fig. 11 demonstrates the spectrum of an audio segment that contains two whistles. The regions with a circle margin correspond to the spectrum when the referee

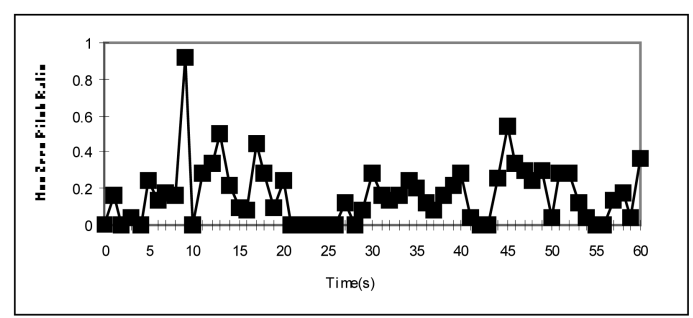

Fig. 10. Nonzero pitch ratio from an audio signal.

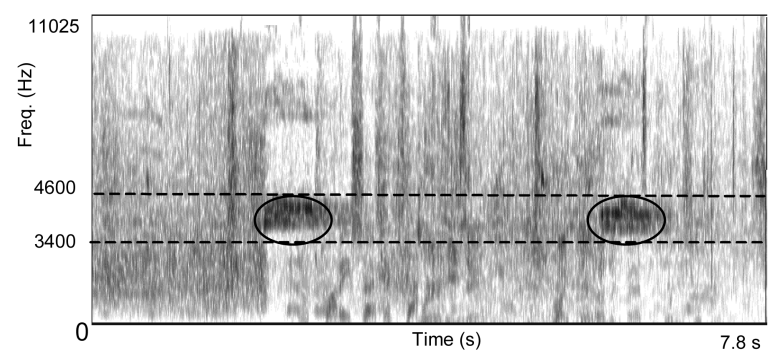

Fig. 11. Spectrum of an audio signal with whistle.

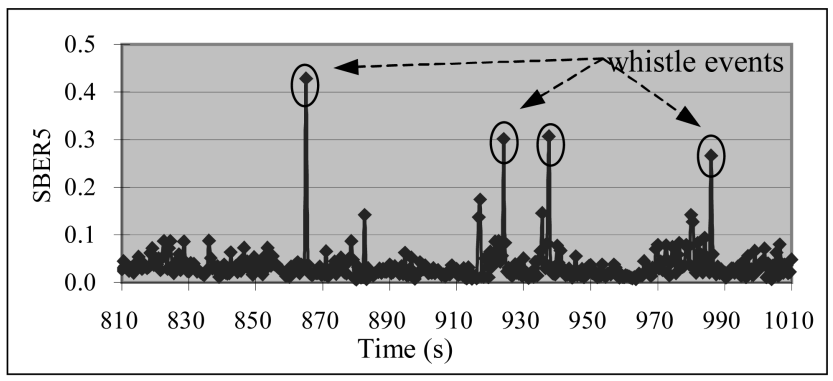

Fig. 12. Segment band energy ratio of the fifth subband from an audio with multiple whistle events.

whistles. One can find that, in frequency regions between $3500 \mathrm{~Hz}$ to $4500 \mathrm{~Hz}$, the energy of a whistle is much higher than others. We then calculate the energy ratio between frequency $3500 \mathrm{~Hz}$ and $4500 \mathrm{~Hz}$ for each audio frame to detect whistles. We split the whole frequency into $B$ subbands. Given audio frame $i$ and subband $j$, we define the band energy ratio (BER) by (7), where $D F T_{i, k}$ is the Discrete Fourier Transformation of the audio frame $i$ and $E$ is the order of DFT coefficients. In our system, the sampling rate for audio signals is $22050 \mathrm{~Hz}$ and $B$ is 12 . Thus, the frequency of the fifth subband is $3675 \sim 4594 \mathrm{~Hz}$. Then, we calculate the segment band energy ratio of the fifth subband $\left(S B E R_{5}\right)$ during a short time period $(0.5 \mathrm{~s})$ by $(8)$, where $A F$ is the total number of audio frames in this period. Fig. 12 shows the results of $S B E R_{5}$ values from a test sports video of about 200 seconds in length. The regions with a circle margin correspond to whistle events. We can then involve some thresholding mechanisms to find out the location of those whistle events.

$$
\begin{gathered}
B E R_{i, j}=\sum_{e=\frac{E}{B}(j-1)}^{\frac{E}{B} j} D F T_{i, e} / \sum_{e=1}^{E} D F T_{i, e}, \\
S B E R_{5}=\frac{1}{A F} \sum_{i=1}^{A F} B E R_{i, 5} .
\end{gathered}
$$




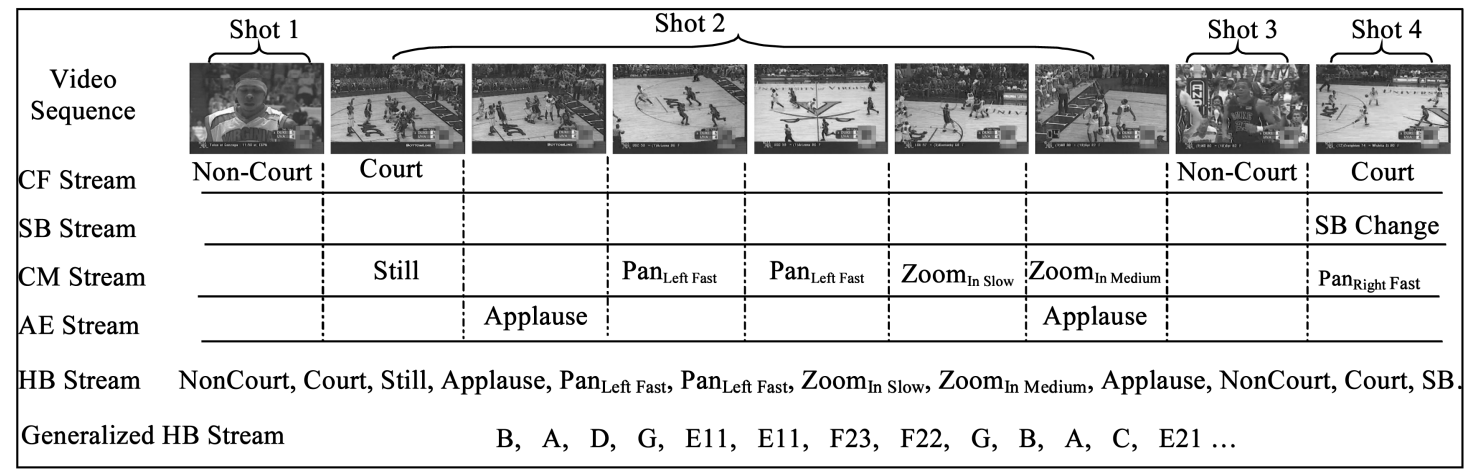

Fig. 13. Video data transformation and generalization.

TABLE 1

A Mapping Table to Generalize Video Data

\begin{tabular}{|c|c|c|c|c|c|c|c|c|c|c|}
\hline \hline \multirow{2}{*}{ Streams } & \multicolumn{2}{|c|}{ CF Stream } & \multicolumn{6}{c|}{ SB Stream } & \multicolumn{6}{c|}{ CM Stream } & \multicolumn{2}{c|}{ AE Stream } \\
\cline { 2 - 13 } & Court & Non-court & SB Change & Still & Pan $_{\text {Left Fast }}$ & Pan $_{\text {Left Med. }}$ & $\ldots$ & Zoom In Slow & Applause & Whistle \\
\hline Generalization & A & B & C & D & E11 & E12 & $\ldots$ & F23 & G & H \\
\hline \hline
\end{tabular}

\section{Association Mining from Video Data}

Generally, there are two types of videos in our daily life: videos with some content structure and videos without any content structure. The former are videos such as movies and news where scenarios are used to convey video content. In [13], [14], we have proposed techniques to mine associations from this type of videos. However, for videos without content structure, e.g., sports videos, associations may still exist where the associations could be characterized as a series of sequentially related actions. For example, in basketball videos, a series of actions, such as Camera pan $\rightarrow$ Camera still $\rightarrow$ Camera zoom-in $\rightarrow$ Applause $\rightarrow$ Scoreboard change, likely appear sequentially, because they usually accompany a goal event. Mining associations from these videos, which do not have content structure, will not only facilitate knowledge acquisition, but also help us in realizing intelligent video management. In this section, we discuss techniques for video association mining, where the definitions and measures for video associations, and the sequential pattern search procedure are extensively studied.

\subsection{Video Data Transformation}

With the techniques in Section 3, the original video sequence is transformed into four separated symbolic streams: court field $(\mathrm{CF})$, camera motion $(\mathrm{CM})$, scoreboard (SB), and audio events (AE), as shown in Fig. 13. Our next step is to conduct mining activities on these streams. To this end, there are two solutions: Treat data streams separately or combine them together as a single stream. Oates and Cohen [36] proposed a mechanism which treats multiple streams separately when conducting the mining activity, where the objective is to find the cooccurrence of the patterns that appear in the multiple streams. However, this method requires that the streams which take part in the mining activity be synchronized, where each stream produces the same amount of symbols in the same amount of time. In our situation, the multiple streams extracted from video data obviously do not satisfy this requirement. Intuitively, combining multiple streams into a single stream appears to be an easier way for data mining purposes because mining from one stream is obviously easier than mining from multiple sources and many research efforts have been conducted to find patterns, e.g., periodic patterns [37], [45], from a data stream. However, we need to guarantee that there is no information loss when combining multiple streams, which means that, after the data combination, we should maintain the original temporal order information of each separate stream in the combined stream. To this end, we adopt the following approach to combine multiple symbolic steams into a single hybrid (HB) stream: 1) For video and audio cues which happen at different time slots, we put all their tags together, with each tag placed at a corresponding place in its original stream. 2) If multiple tags happen at the same time, e.g., a scoreboard change and a camera motion happen at the same time, we use the same order to combine them in all situations, e.g., a scoreboard change always precedes a camera motion. An example of video data transformation is shown in Fig. 13, where information from four separate streams is combined to form a hybrid stream. ${ }^{2}$ With such a mechanism, the temporal order information in each separate stream is well maintained in the transferred hybrid stream and combining multiple streams into a single stream will not lose information for effective association mining from data streams.

We have adopted a hierarchical camera motion description in Fig. 8, so we have to generalize an $H B$ stream for multilevel association mining. Our generalization is accomplished by assigning a symbol to each type of tag, as shown in Table 1. For events with a hierarchy, we generalize them into a set of characters with each character indicating a state. For example, for "E12," "E" denotes camera pan, "1"

2. We mark only one CF tag for each video shot, which is placed at the beginning of the shot, because a shot either belongs to the court field or not. Inside each shot, we will analyze its content and explore other video and audio cues. This is the reason that some video shots receive several tags, as shown in shot 2 of Fig. 13. This is different from the statements in Section 3.1, where only one key-frame is extracted from each shot to classify a video shot into a noncourt shot or a court shot. 
indicates the panning direction, and " 2 " represents the motion magnitude. In Fig. 13, the last row gives a generalized $H B$ stream.

\subsection{Definitions and Terminology}

Based on the above observations, we define a video association as a sequential pattern with $\left\{X_{1} . . X_{i} . . X_{L} ; X_{i}^{t}<\right.$ $X_{j}^{t}$ for any $\left.i<j\right\}$, where $X_{i}$ is a video item (see Definition 1 below), $L$ denotes the length of the association, $X_{1} \cap . . \cap X_{i} . . \cap X_{L}=\oslash, X_{i}^{t}$ denotes the temporal order of $X_{i}$, and $X_{i}^{t}<X_{j}^{t}$ indicates that $X_{i}$ happens before $X_{j}$. For simplicity, we use $\{X\}$ as the abbreviation for a video association.

Generally, two measures (support and confidence) have been used to evaluate the quality of an association. However, these measures do not consider temporal information of the items in the association. For video associations, the temporal distance (see Definition 5 below) between neighboring items implies some useful information: The smaller the temporal distance between neighboring items, the larger is their correlation. For example, if two neighboring shots contain applause and scoreboard change, respectively, we naturally believe that they are correlated. However, the applause that happens several shots (e.g., three more shots) before the scoreboard change rarely indicates any correlation between them. That is, for associations with a large temporal distance between neighboring items, their items usually have a weaker correlation and, therefore, can imply almost no knowledge. Accordingly, instead of using the traditional support measure, we adopt a temporal support (TS) to evaluate the support of each association. Moreover, several other definitions are also given below:

1. A video item is a basic unit in association mining. In this paper, it denotes a symbolic tag acquired from video processing techniques, i.e., a symbolic unit in the hybrid video stream.

2. An L-ItemAssociation is an association that consists of $L$ sequential items. For example, "AB" is a 2-ItemAssociation and "ABC" is a 3-ItemAssociation.

3. An ItemSet is an aggregation which consists of video associations. More specifically, an L-ItemSet is an aggregation of all L-ItemAssociations, each of which is an L-ItemAssociation.

4. L-LItemSet is an aggregation of all L-ItemAssociations whose temporal support (see Definition 7 below) is no less than a given threshold.

5. Given a transformed hybrid video stream, the temporal distance $(T D)$ between two items is the temporal identification difference of the shots that contain these two items. For example, in the hybrid stream demonstrated in Fig. 14, the first time the pattern $\{A B\}$ appears, their temporal distance $T D\{A B\}$ is 0 because they happen in the same shot. The second time $\{A B\}$ appears, $T D\{A B\}$ equals 1 because A and $B$ happen in two neighboring shots and the temporal identification difference between the neighboring shots is 1 .

6. The temporal distance threshold (TDT) specifies the upper bound that the temporal distance must comply with, i.e., no larger than this threshold. Take the pattern $\{A B\}$ in Fig. 14, for example, when $T D T=1, T D\{A B\}=2$ will not satisfy because $T D\{A B\}=2$ is larger than the given $T D T$ value.

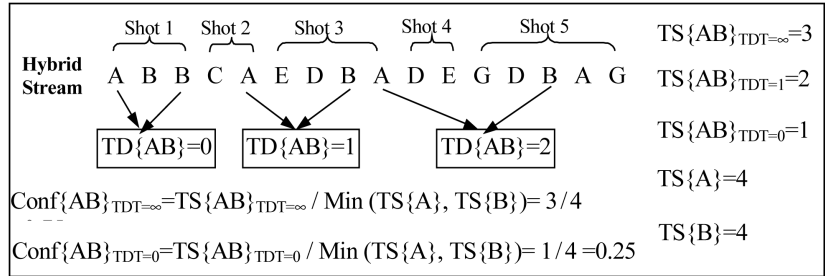

Fig. 14. Example of video association evaluation in terms of temporal support and confidence

7. Given a temporal distance threshold (TDT) $T D T=T$, the temporal support (TS) of an association $\left\{X_{1} \ldots X_{L}\right\}$ is defined as the number of times this association appears sequentially in the sequence. In addition, each time this association appears, the temporal distance between any two neighboring items of the association should satisfy the given $T D T$ (i.e., no more than $T$ shots). In Fig. 14, when $T D T=\infty$ (i.e., ignoring the temporal distance), the temporal support for $\{A B\}$ is $T S\{A B\}=3$. However, when we set $T D T=1, T S\{A B\}$ becomes 2 because the last time $\{A B\}$ appears, its temporal distance $(T D\{A B\}=2)$ does not satisfy the given $T D T$. It is obvious that the smaller the $T D T$, the stronger the semantic correlations among the mined associations are.

Given $T D T=T$, the confidence of an association $\left\{X_{1}, ., X_{L}\right\}$ is defined as the ratio between the temporal support of $\{X\}$ (when $T D T=T$ ) and the number of maximal possible occurrences of the association $\{X\}$. Because the maximal possible occurrences of the association are determined by the number of occurrences of the item with the minimal support, the confidence of the association is defined by (9). The examples of the confidence evaluation have been provided in Fig. 14, where different $T D T$ values result in different confidences for the same association. The larger the confidence value, the more confidently the association holds:

$$
\operatorname{Conf}\{X\}_{T D T=T}=T S\{X\}_{T D T=T} / \operatorname{Min}\left(T S\left(X_{1}\right), . ., T S\left(X_{L}\right)\right) .
$$

\subsection{Video Association Mining}

\subsubsection{Multilevel Associations}

We have introduced a hierarchy in Fig. 8 (which can also be interpreted as a taxonomy) to characterize camera motions. When a taxonomy exists, the supports of associations at lower levels are lower than associations at higher levels. Accordingly, some solutions have been proposed to mine multilevel associations [38]. The motivation behind these algorithms is simple and intuitive: For all hierarchical items, their ancestors at higher levels are added into data sets and a data mining algorithm is executed on new data sets for multiple times to mine multilevel associations. As shown in Table 2, given a generalized $H B$ sequence in Tables $2 a, 2 b$, and $2 c$, show the 1-ItemSet at level 1 and level 2, respectively. As we can see, only the descendants of the large ItemSet at level 1 are considered as candidates for level 2 large 1-ItemSet. 
TABLE 2

Multilevel Association Mining: (a) A Generalized HB Sequence, (b) 1-ItemSet at Level 1, and (c) 1-ItemSet at Level 2

\begin{tabular}{|c|}
\hline \hline Generalized HB sequence \\
\hline F11, A, E13, C, F22, F23 \\
E12, D, F21, A, E23, C, A, \\
F13 \\
\hline
\end{tabular}

(a)

\begin{tabular}{|c|c|}
\hline \hline 1-ItemSet & Suppor \\
\hline$\{\mathrm{A}\}$ & 3 \\
$\{\mathrm{E} * *\}$ & 3 \\
$\{\mathrm{~F} * *\}$ & 5 \\
\hline \hline
\end{tabular}

(b)

\begin{tabular}{|c|c|}
\hline \hline 1-ItemSet & Support \\
\hline$\{\mathrm{A}\}$ & 3 \\
$\{\mathrm{E} 1 *\}$ & 2 \\
$\{\mathrm{~F} 1 *\}$ & 2 \\
\hline
\end{tabular}

(c)

\subsubsection{The Mining Algorithm}

Our video association mining algorithm consists of the following phases:

1. Transform. This phase adopts various techniques to explore visual and audio cues and transforms video data into a relational data set $D$.

2. L-LItemSet. In this phase, we mine video associations with various levels and lengths. We first find an L-ItemSet and then use the L-ItemSet and userspecified thresholds to find L-LItemSet. We iteratively execute this phase until no more nonempty L-LItemSet can be found.

3. Collection and Postprocessing. This phase collects and postprocesses video associations for different applications.

We have discussed techniques for Phase 1 and Phase 3 directly relates to applications of video associations, which is trivial from the data mining point of view. Therefore, we focus on Phase 2 only, where its main procedure is shown in Fig. 15. Throughout this section, we use the notions that $l$ denotes the level of associations (the maximal level of associations max_level is 3 in our system) and $D[l]$ represents the filtered data set at level $l . I[l, k]$ and $L[l, k]$ are the aggregations of $k$-ItemSet and $k$-LItemSet at level $l$, respectively. $\{\mathrm{X}\} . \mathrm{Item}_{\mathrm{k}}$ means the $k$ th item of the association $\{X\}$.

Basically, Phase 2 consists of two stages: 1) In the first stage, the algorithm filters the data set at level $l$ and uses the filtered data set $D[l]$ to construct 1-ItemSet and 1-LItemSet at level $l$, as shown on lines 2 to 4 in Fig. 15. 2) Then, the algorithm uses the constructed 1-LItemSet and the candidate generation procedure (Fig. 16) to progressively mine $k$-ItemAssociations, $k=2,3, \ldots$, at level $l$, until the constructed $k$-LItemSet at level $l$ is empty. Then, the algorithm turns to next level $l+1$ and mines associations at this level.

As shown in Fig. 15, for each level $l$, we first filter the data set $D$, Filter_Dataset $(D, l)$, to process items that are no larger than level $l$. For example, when $l=2$, this procedure filters items $\{E 13, E 12\}$ as $\{E 1, E 1\}$ and the higher the level, the more subtle the filtered item is. The filtered sequence is put into a new data set $D[l]$. We then use $D[l]$ to generate 1-ItemAssociations at level $l$ (denoted by $I[l, 1]$ ) by using function $\operatorname{Get}_{1} 1 \_$ItemSet $(D[l], l)$. We use the generated 1-ItemSet and the user specified minimal support minSup $[l]$ to generate 1-LItemSet at level $l$ (denoted by $L[l, 1]$ ) with procedure Get_1_LItemSet $(D[l], I[l, 1], \operatorname{minSup}[l])$. The generated 1-LItemSet consists of associations in 1-ItemSet which satisfy the user-specified minimal support minSup $[l]$. Because 1-ItemAssocitions do not involve any temporal distance, we ignore TDT when constructing the 1-LItemSet. We then use the generated 1-LItemSet at level $l$ to mine associations with larger lengths. This is facilitated by adopting an Apriori-like algorithm which uses multiple passes to generate candidates and evaluate their supports.

In each pass, we use the LItemSet from the previous pass to generate the candidate ItemSet and then measure the temporal support of generated candidates by making a pass over the database $D[l]$. At the end of the pass, the support of each candidate is used to determine the frequent ItemSet.

Candidate generation for each pass is similar to the method in [12]. It takes the set of all $k-1$-ItemAssociations in $L[l, k-1]$ and all their items as input and works as shown in Fig. 16. The items in $L[l, k-1]$ first join together to form new candidates. To this end, for any two distinct $k-$ 1-ItemAssociations $\{p\}$ and $\{q\}$ in $L[l, k-1]$, if their first $k-2$ items are the same (as shown on line 3 in Fig. 16), we will generate a new $k$-ItemAssociation $\{X\}$. The first $k-2$ items of $\{X\}$ are the same as that of $\{p\}$ and the $k-1$ th and $k$ th items of $\{X\}$ are the $k-1$ th item of $\{p\}$ and $\{q\}$, respectively (as shown on line 5 in Fig. 16). Then, $\{X\}$ is

Procedure VAMining ()

Input: (1) Hybrid data steam D; (2) max. association level max_level; and (3) TDT and minimal support and confidence in selecting associations at different levels $\operatorname{minSup}[l], \min \overline{C o n f}[l], l=1, \ldots$, max_level.

Output: Mined multi-level video associations
(1) For $(l=1 ; l \leq$ max_level; $l++)$
(2) $\quad D[l]=$ Filter_Dataset $(D, l)$;
(3) $\quad I[l, 1]=$ Get_l_ItemSet $_{(}(D[l], l)$
(4) $\quad L[l, 1]=G e t \overline{1}$ LItemSet $(D[l], I[l, 1], \operatorname{minSup}[l])$
(5) $\quad$ For $(k=2 ; L[l, k-1] \neq \emptyset ; k++)$
(6) $\quad I[l, k]=$ CandidateGeneration $(L[l, k-1])$
(7) $\quad L[l, k] \leftarrow \Phi$
(8) For each $k$-ItemAssociation $\{X\}$ in $I[l, k],\{X\} \in I[l, k]$
(9) $\quad \operatorname{TS}\{X\}_{T D T}=$ Calculate_TS $(D[l], T D T)$
(10) $\quad \operatorname{Conf}\{X\}_{T D T}=\operatorname{TS}\{\mathrm{X}\}_{\mathrm{TDT}}^{-} \operatorname{Min}\left(T S\left\{X_{l}\right\}, \ldots, T S\left\{X_{k}\right\}\right)$
$/ /$ mine associations at various levels
$/ /$ process items that are no larger than level $l$
$/ /$ find 1-ItemSet at level $l$
$/ /$ find 1 -LItemSet at level $l$
$/ /$ mine associations with different lengths
//generate candidates, see Fig. 16
// initialize $k$-LItemSet
//evaluate each generated candidate
// calculate temporal support of $X$
// calculate confidence of $X$
(11) $\quad L[l, k] \leftarrow L[l, k] \cup\{\mathrm{X}\} \mid\{X\} \in I[l, k], \operatorname{TS}\{X\}_{T D T} \geq \operatorname{minSup}[l] \& \operatorname{Conf}\{X\}_{T D T} \geq \min \operatorname{Conf}[l]$
//select associations with their temporal support and confidence larger than the given thresholds
Endfor

Fig. 15. Pseudocode for multilevel video association mining. 
Procedure CandidateGeneration()
Input: $L[l, k-1] \quad / / k$-1 large item set at level $l$ Output: $I[l, k]$
(1). $\quad I[l, k] \leftarrow \Phi$
(2). For any two $k$-1-ItemAssocations $\{p\}$ and $\{q\}$ in $L[l, k-1]$
$/ / k$ item set at level $l$
(3). $\quad$ If $(\{p\} \neq\{q\})$ and $\left(\{p\}\right.$. Item $_{1}=\{q\}$. Item $_{1} \& . . \&\{p\}$. Item $_{k-2}=\{q\}$. Item $\left._{k-2}\right)$
(4). $\quad:$ Generate a $k$-ItemAssociation $\{X\}$ such that:
(5). $\quad\{X\} \cdot$ Item $_{l}=\{p\} . \operatorname{Item}_{1}, \& \ldots \&,\{X\} \cdot \operatorname{Item}_{k-2}=\{p\} \cdot \operatorname{Item}_{k-2},\{X\} \cdot \operatorname{Item}_{k-1}=\{p\} . \operatorname{Item}_{k-1},\{X\} . \operatorname{Item}_{k}=\{q\} . \operatorname{Item}_{k-1}$
(6). $\quad$ End If
(7). End For
(8). Delete any member $\{X\} \in I[l, k]$ such that some $k$-1-ItemAssociation of $\{X\}$ is not in $L[l, k-1]$.

Fig. 16. Pseudocode for candidate generation.

TABLE 3

An Example of Video Association Mining, where $\{X\}_{S}^{C}$ Indicates an Association

\begin{tabular}{|c|c|c|c|c|c|}
\hline $\begin{array}{c}\text { Hybrid Stream } D \\
\text { (left to right, top } \\
\text { to bottom) }\end{array}$ & $(T D T=1)$ & $\begin{array}{c}\text { 3-LItemSet } \\
(T D T=\infty)\end{array}$ & $\begin{array}{c}\text { Candidate 4- } \\
\text { ItemSet (after join) }\end{array}$ & $\begin{array}{c}\text { 4-ItemSet } \\
\text { (after pruning) }\end{array}$ & $\begin{array}{c}\text { 4-LItemSet } \\
(T D T=\infty)\end{array}$ \\
\hline A B C B C & $\{A B\}_{4}^{1.0},\{A C\}_{3}^{0.8},\{A D\}_{1}^{0.3},\{B A\}_{2}^{0.5}$, & $\{A B\}_{4}^{1.0},\{A C\}_{3}^{0.8},\{A D\}_{3}^{1.0},\{B A\}_{3}^{0.8}$, & $\{A B C\}_{3}^{0.8},\{A B D\}_{3}^{1.0}$ & $\{A B C D\}$ & $\{A B C D\}$ \\
D C A C B & $\{B\}_{1}^{0.3},\{B C\}_{4}^{0.7},\{B D\}_{3}^{1.0},\{C B\}_{4}^{0.7}$, & $\{B B\}_{3}^{1.0},\{B C\}_{4}^{0.7},\{B D\}_{3}^{1.0},\{C B\}_{4}^{0.7}$, & $\{A C B\}_{3}^{0.8},\{A C D\}_{3}^{1.0}$ & $\{A B D C\}$ & $\{A C B D\}$ \\
C A B D A & $\{C C\}_{2}^{0.7},\{C D\}_{2}^{0.7},\{D B\}_{2}^{0.7}$ & $\{C C\}_{3}^{1.0},\{C D\}_{3}^{1.0},\{D B\}_{3}^{1.0}$ & $\{B C D\}_{3}^{1.0},\{C D B\}_{3}^{1.0}$ & $\{A C D B\}$ & $\{A C D B\}$ \\
B C D B E & $\{A B C D\}_{3}^{1.0}$ \\
\hline
\end{tabular}

$X$ denotes the items of the association, $S$ and $C$ indicate the temporal support, and confidence of the association, respectively (for simplicity, we assume each video shot has only one symbolic tag and the HB stream has only one level).

taken as a candidate and put in $I[l, k]$. We iteratively repeat the same procedure until all elements in $L[l, k-1]$ have been evaluated. After that, we prune out the candidates in $I[l, k]$ whose subsequences are not in $L[l, k-1]$ because, if a subsequence of an association is not frequent, this association will not be frequent neither. All remaining candidates are taken as associations in $I[l, k]$. Table 3 provides an example of candidate generation, where the fourth column gives the 3-LItemSet and the fifth column is the join results (candidates) from the 3-LItemSet. After pruning out sequences whose subsequences are not in the 3-LItemSet, the sequences shown in the sixth column will be left. For example, $\{\mathrm{ABDC}\}$ is pruned out because its subsequence $\{\mathrm{BDC}\}$ is not in the 3-LItemSet.

\subsubsection{Search Patterns from Hybrid Stream with Constraints}

To mine video associations, the most important procedure is to search the appearances of the candidate pattern in the data stream, and this problem is complicated by users' constraint on the temporal distance (TDT) between items of the pattern. For example, with the HB stream in Fig. 17a, when searching the appearance for pattern $\{A E F B G\}$, many other approaches [37], [39] usually adopt a sliding window (e.g., $w_{1}$ and $w_{2}$ in Fig. 17a) to evaluate whether the pattern appears in the window or not. Such a windowing procedure has two obvious disadvantages: 1) Users have no control with the temporal distance between the items of the pattern, i.e., this approach ignores the temporal distances in the pattern, and 2) users have to well define the width of the window, otherwise the pattern may never fall into any window.

Accordingly, we need to design a new search mechanism by considering the temporal distance between neighboring items of the pattern. The simplest solution for this problem is to adopt a waiting-and-matching [46] method: We start from the first item of the pattern $\{A E F B G\}$ and scan the data stream until the certain item appears; at any state, if the temporal distance between items violates the $T D T$, the search procedure restarts. In Fig. 17a, "choice 1 " of $\{A E F B G\}$ represents the results from this method. This approach, however, could miss targets if the user specifies a relatively small $T D T$. In Fig. 17a, if we set $T D T=2$, the waiting-andmatching mechanism will fail to find the pattern because the temporal support between " $B G$ " in "choice 1 " is 3 , which is larger than $T D T=2$. However, there are other choices that

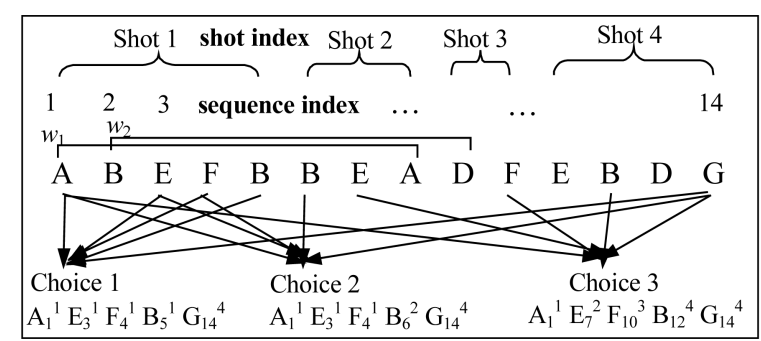

(a)

\begin{tabular}{|c|c|c|}
\hline $\begin{array}{r}\text { Objectiv } \\
\text { Pattern }\end{array}$ & $\begin{array}{lc}\text { ive } & \text { Initialize } \\
\text { n } & \text { Lists }\end{array}$ & $\begin{array}{c}\text { Status at } \\
\mathrm{F}_{10}{ }^{3}\end{array}$ \\
\hline A: & $O_{l}=\Phi \Rightarrow \quad\left\{\mathrm{A}_{1}^{1}\right\}$ & $\Rightarrow\left\{\mathrm{A}_{1}{ }^{1}, \mathrm{~A}_{8}{ }^{2}\right\} \Rightarrow$ \\
\hline E: & $O_{2}=\Phi \Rightarrow\left\{\mathrm{E}_{3}^{1}\right\}$ & $\Rightarrow\left\{\mathrm{E}_{3}{ }^{1}, \mathrm{E}_{7}{ }^{2}\right\} \Rightarrow$ \\
\hline $\mathrm{F}$ : & $O_{3}=\Phi \Rightarrow\left\{\mathrm{F}_{4}{ }^{1}\right\}$ & $\Rightarrow\left\{\mathrm{F}_{4}{ }^{1}, \mathrm{~F}_{10}{ }^{3}\right\} \Rightarrow$ \\
\hline B: & $O_{4}=\Phi \Rightarrow\left\{\mathrm{B}_{5}{ }^{1}\right.$, & $\Rightarrow\left\{\mathrm{B}_{5}{ }^{1}, \mathrm{~B}_{6}{ }^{2}\right\}$ \\
\hline G: & $O_{5}=\Phi \Rightarrow$ & $\Rightarrow$ \\
\hline
\end{tabular}

(b)

Fig. 17. Search candidates from a hybrid stream, where $X_{i}^{j}$ represents the index information of the item $X$ ( $j$ means in which shot the item appears and $i$ indicates the order of the item in the stream). (a) An example of hybrid stream. (b) Search procedure (TDT=2). 
$\{A E F B G\}$ actually satisfies $T D T=2$, e.g., "choice 2 " and "choice3."

Motivated by the above observations, we propose a new algorithm for searching special patterns from data stream with constraints. The intuitive idea behind this scheme is to push an item backward as much as we can (without violating the $T D T$ ), so we can maximize the possibility that, under the constraint of $T D T$, the pattern may appear in the stream. The algorithm consists of following major steps:

1. Given a pattern $\left\{X_{1}, X_{2} . ., X_{L}\right\}$, a hybrid stream $D$, and a user-specified $T D T$, we call the objective pattern in $D\left\{X_{1}, X_{2} . ., X_{L}\right\}$. For each item $X_{i}$ in the objective pattern, we construct a list $O_{i}$ to record the appearances of $X_{i}$ in $D$ and initialize the list with $O_{1} \leftarrow \Phi, \ldots, O_{i} \leftarrow \Phi ;, \ldots, O_{L} \leftarrow \Phi ;$.

2. Starting from the first item of the objective pattern, for each item $X_{i}, i=1, . . L$, we search the first appearance of $X_{i}$ from $D$ (and ignore the appearance of any other item $X_{j}, j>i$ ). If item $X_{i}$ appears in $D$, we put the index (sequence index and shot index) of the appearance into the list $O_{i}$. As demonstrated in Fig. 17b, $O_{1} \leftarrow O_{1} \cup A_{1}^{1}$; $O_{2} \leftarrow O_{2} \cup E_{3}^{1}$, and so on. This procedure continues until all items $X_{i}, i=1, . ., L$, have at least one member in their list $O_{i}, i=1, . ., L$.

3. When searching the appearance of the current item $X_{i}$, if any former item (including $X_{i}$ itself) $X_{j}, j \leq i$ appears in $D$ again, we put the index of $X_{j}$ in the list $O_{j}$ as long as the appearance of $X_{j}$ satisfies the $T D T$. As shown at "status in $B_{6}^{2 \text { " }}$ in Fig. 17b, when searching for the appearance of " $G$," another " $B$ " comes. Denote $O_{i}^{k}$ by the $k$ th member in the list $O_{i}$, and $T_{i}$ by the number of members in $O_{i}$, so $O_{i}^{T_{i}}$ is the last member in $O_{i}$. To evaluate whether the appearance of $X_{j}$ satisfies the constraint of $T D T$, we calculate two measures: a) the temporal distance between $X_{j}$ and the latest appearance of its neighboring item $O_{j-1}^{T_{j-1}}$, $T D\left(X_{j}, O_{j-1}^{T_{j-1}}\right)$ and $\left.\mathrm{b}\right)$ the temporal distance between the current location of $X_{j}$ and the last member in $O_{i-1}, T D\left(X_{j}, O_{i-1}^{T_{i-1}}\right)$. If $T D\left(X_{j}, O_{j-1}^{T_{j-1}}\right) \leq T D T$, we add the index of $X_{j}$ into its list $O_{j}$ and continue the procedure; otherwise, $O_{j}$ remains unchanged. Meanwhile, if $T D\left(X_{j}, O_{i-1}^{T_{i-1}}\right)>T D T$, it will indicate that, even if we assume the item $X_{i}$ does appear at the current location of $X_{j}$, the temporal distance with its neighboring item $X_{i-1}$ still violates the constraint of $T D T$, so there is no need to search the appearance of $X_{i}$ any further. We will restart searching the appearance of the objective pattern from the location of the last member in $O_{1}$. Meanwhile, all lists should be initialized with $O_{1} \leftarrow \Phi, . ., O_{i} \leftarrow \Phi, . . O_{L} \leftarrow \Phi$.

4. As long as the lists of all items $\left(O_{1}, O_{2}, \ldots, O_{L}\right)$ have at least one member, we cease the current search procedure because an appearance of the pattern have been located so far. As shown at "status at $G_{14}^{4} \ldots "$ in Fig. $17 \mathrm{~b}$, we will start from the last member in $O_{L}$ (actually, there is only one member in $O_{L}$ ), denote it by $O_{L}^{*}$, and check all members in $O_{L-1}$ in an inverse order (backward) to find the member that appears before $O_{L}^{*}$ and has the smallest temporal distance with $O_{L}^{*}$. We denote this member by $O_{L-1}^{*}$ and then find the member from $O_{L-2}$ that appears before $O_{L-1}^{*}$ and has the smallest temporal distance with $O_{L-1}^{*}$. We repeat the same procedure until the appearances of all items have been located. The sequence $\left\{O_{1}^{*}, . ., O_{L}^{*}\right\}$ will provide actual locations of the pattern, as shown in Fig. 17b. Then, we initialize all lists with $O_{1} \leftarrow \Phi, . ., O_{i} \leftarrow \Phi, . . O_{L} \leftarrow \Phi$ and restart to locate the next appearance of the pattern from the location next to $O_{L}^{*}$.

As shown in Fig. 17 , no matter what $T D T$ value $(1,2$, or 3) users specify, our algorithm will exactly locate only one location for $\{A E F B G\}$, which is "choice 3." However, with the waiting-and-matching approach, only "choice 1" could be found and, if we set $T D T=1$, it will miss the appearance of the pattern because, in this case, "choice 1" does not satisfy the TDT. Therefore, our algorithm has a higher accuracy than the waiting-and-matching mechanism. And, because we only scan stream $D$ once, the complexity of the algorithm is $O(N)$ for one objective pattern, where $N$ is the length of $D$.

\section{Video Association Classification}

To apply video associations in video indexing, we need to classify each association into a corresponding category (event) and use detected events to construct video indices. Some research efforts have addressed the problem of association rule classification, but little literature has been found on classifying sequential associations. We adopt the nearest neighbor search-based strategy as follows: We first mine associations from training videos. For each association, we manually go through the training data to evaluate what types of events associate with the appearance of this association. We count the number and the types of events from all appearances and select the event with the largest number to label the association. Accordingly, each association will receive one class label. For each association, $\{X\}$, in the test set, we calculate its distance with associations in the training set and the class label of the association in the training set which has the smallest distance with $\{X\}$ is used to label $\{X\}$. In the case that multiple associations have the same smallest distance with $\{X\}$, all their class labels are used to label $\{X\}$. To calculate the distance between sequential associations, we take the temporal order and the length of the associations into consideration and use the Longest Common Subsequence (LCS) [40] between two associations to evaluate the association distances.

Given two associations, assuming $\{X\}^{1}=\left\{X_{1}, \ldots, X_{P}\right\}$ denotes the association with a length, $P$, and the other association is denoted by $\{X\}^{2}=\left\{X_{1}, \ldots X_{Q}\right\}$ with length $Q$. For example,

$$
\{X\}^{1}=\{\mathrm{A}, \mathrm{B}, \mathrm{E}, \mathrm{D}, \mathrm{G}\}
$$

and $\{X\}^{2}=\{\mathrm{B}, \mathrm{A}, \mathrm{E}, \mathrm{G}, \mathrm{A}, \mathrm{D}\}$. The Dynamic Programming [40] has $O(P Q)$ time complexity and space requirement to find the largest common subsequence between $\{X\}^{1}$ and $\{X\}^{2} .{ }^{3}$ Then, the distance between $\{X\}^{1}$ and $\{X\}^{2}$ is

3. In the example above, there are two LCS subsequences $\operatorname{LCS}\left\{\{\mathrm{X}\}^{1},\{\mathrm{X}\}^{2}\right\}=\{\{\mathrm{A}, \mathrm{E}, \mathrm{G}\},\{\mathrm{B}, \mathrm{E}, \mathrm{G}\}\}$. 
TABLE 4

Video Shot Clustering Results

\begin{tabular}{c|ccc|ccc}
\hline \hline \multirow{2}{*}{ Video Source } & \multicolumn{3}{|c|}{ Group $_{A}$} & \multicolumn{3}{c}{ Group $_{B}$} \\
\cline { 2 - 7 } & $S^{\prime} N_{A}$ & $F_{A}$ & Accuracy $_{A}$ & STN $_{B}$ & $F_{B}$ & Accuracy $_{B}$ \\
\hline LAL vs HOU & 414 & 388 & 0.937 & 312 & 290 & 0.929 \\
\hline DUKE vs UVA & 332 & 289 & 0.870 & 282 & 253 & 0.897 \\
\hline TOR vs BOS & 355 & 329 & 0.927 & 314 & 279 & 0.888 \\
\hline HOU vs DEN & 474 & 432 & 0.911 & 306 & 268 & 0.876 \\
\hline Average & 1575 & 1438 & 0.914 & 1214 & 1090 & 0.898 \\
\hline \hline
\end{tabular}

defined by (10), where $\left|\operatorname{LCS}\left\{\{\mathrm{X}\}^{1},\{\mathrm{X}\}^{2}\right\}\right|$ represents the length of the largest common subsequence:

$$
\operatorname{SeqAssocD}\left\{\{X\}^{1},\{X\}^{2}\right\}=1-\frac{\left|L C S\left\{\{X\}^{1},\{X\}^{2}\right\}\right|}{\operatorname{Min}(P, Q)},
$$

Actually, this distance is determined by the maximal number of sequentially matched items between the associations $\{X\}^{1}$ and $\{X\}^{2}$. The larger the number, the smaller their distance is.

\section{EXPERIMENTAL RESULTS}

In this section, we present the results of an extensive performance analysis we have conducted to 1) evaluate the video processing techniques in Section 3,2) evaluate the video association mining and association-based indexing algorithms in Sections 4 and 5, and 3) analyze the performance of our knowledge-based indexing framework. We evaluate our algorithms with eight basketball videos (NBA and NCAA) captured from ESPN and Fox and all commercials in the videos are removed.

\subsection{Video Preprocessing Results}

For the sake of conciseness, we only present results of shot classification and audio events detection. One can refer to other references [34], [35] for performances of video text and camera motion detection.

\subsubsection{Video Shot Clustering and Classification}

In Section 3.1, we have clustered video shots into groups and classified each group into two categories: The first consists of court field shots $\left(\right.$ Group $\left._{A}\right)$ and the second consists of noncourt field shots $\left(\right.$ Group $\left._{B}\right)$. To evaluate the performance of our clustering algorithm, we manually classify video groups into these two categories and then count the number of shots in Group $A$ which do belong to Group $_{A}$ (i.e., shots which contain a court field and are clustered into a group which mainly consists of court field shots) and denote this number by $F_{A}$. We also count the number of shots in Group $_{B}$ which do belong to Group G $_{B}$ and denote this number by $F_{B}$. The clustering accuracy of each category is defined by (11), where $S T N_{A}$ and $S T N_{B}$ represent the number of shots contained in groups which belong to Group $_{A}$ and Group $_{B}$, respectively:

$$
\text { Accuracy }_{A}=F_{A} / S T N_{A} ; \quad \text { Accuracy }_{B}=F_{B} / S T N B .
$$

To evaluate the performance of the group classification, we count the number of groups that belong to Group $_{A}$ and Group $_{B}$ and denote these two numbers by GPNum $A$ and $G P N u m_{B}$, respectively. Also, we denote the number of
TABLE 5

Video Shot Classification Results

\begin{tabular}{c|ccc|ccc}
\hline \hline \multirow{2}{*}{ Video Source } & \multicolumn{3}{|c|}{ Group $_{A}$} & \multicolumn{4}{c}{ Group $_{B}$} \\
\cline { 2 - 7 } & $G_{A}$ & GF $_{A}$ & GAccuracy $_{A}$ & $G^{\prime} N_{B}$ & GF $_{B}$ & GAccuracy $_{B}$ \\
\hline LAL vs HOU & 24 & 21 & 0.875 & 33 & 28 & 0.848 \\
\hline DUKE vs UVA & 19 & 17 & 0.895 & 27 & 23 & 0.852 \\
\hline TOR vs BOS & 23 & 20 & 0.870 & 28 & 23 & 0.821 \\
\hline HOU vs DEN & 25 & 21 & 0.840 & 36 & 30 & 0.833 \\
\hline Average & 91 & 79 & 0.869 & 124 & 104 & 0.839 \\
\hline \hline
\end{tabular}

groups which belong to Group $_{A}$ and are correctly classified as rroup $_{A}$ by $G F_{A}$ and, similarly, the number of groups belonging to Group $_{B}$ and are correctly classified as $\operatorname{Group}_{B}$ is denoted by $G F_{B}$. The accuracy of group classification is defined by (12).

$$
\begin{aligned}
& \text { GAccuracy }_{A}=G F_{A} / G P \text { Num }_{A} ; \\
& \text { GAccuracy }_{B}=G F_{B} / G P N u m_{B} .
\end{aligned}
$$

We perform experiments on four videos and present their results in Table 4 . The results in Table 4 indicate that the proposed clustering algorithm is very successful on basketball videos. On average, the accuracy of Group $_{A}$ and Group $_{B}$ are 0.914 and 0.898 , respectively, that is, only a small percentage of shots are falsely clustered into the wrong cluster. We have used this algorithm to test other types of videos, e.g., movies, news, and medical videos, and found the results from the basketball videos are remarkably better. One reason is that a basketball video is usually captured from cameras at different locations and views of the same stadium. Hence, the proposed features and distance functions can efficiently address visual differences. As shown in Fig. 5, court field shots are likely to be merged into groups, with each group being characterized by the camera from a certain view.

With the results in Table 5, we can find that the dominant color can be used to classify court shots in basketball videos. In all Group $A$ groups, the accuracy is satisfactory because court field shots do exhibit a distinct dominant color. However, we notice that more $\operatorname{Group}_{B}$ groups are falsely classified as Group $_{A}$. The reason is that these groups likely contain some specially edited shots. For example, a tag "look back" indicates that the subsequent shots are a review and the "tag" shot likely contains the "yellow" dominant color, as shown on the fourth row of Fig. 5. Fortunately, the number of these types of shots is very limited and, on the other hand, these shots do not have other valuable visual or audio cues. Even if we falsely classify them as court shots, they won't bring much trouble into our algorithms.

\subsubsection{Salient Audio Event Detection}

To evaluate the performance of the proposed salient audio event detection in Section 3.4, we apply our methods on one NBA video (which lasts about 70 minutes). We manually go through the video to evaluate each detected audio event and present the results in Table 6 . One can find that, by adopting the proposed pitch feature, we can distinguish applause from other events with a satisfactory result, where the average precision and recall are 80.6 percent and 76.3 percent, respectively. However, the precision of the whistle detection algorithm is pessimistic ( 52.5 percent), although the recall of this method is very successful with 
TABLE 6

Salient Audio Events Detection Results

\begin{tabular}{c|c|c|c}
\hline \hline \multicolumn{2}{|c|}{} & Applause & Whistle \\
\hline \multicolumn{2}{c|}{ Actual Segments } & 76 & 66 \\
\hline \multirow{2}{*}{$\begin{array}{c}\text { Detection } \\
\text { Results }\end{array}$} & Correct & 58 & 64 \\
\cline { 2 - 4 } & False Alarm & 14 & 58 \\
\cline { 2 - 4 } & Total & 72 & 122 \\
\hline \multicolumn{2}{c|}{ Precision } & $58 / 72=80.6 \%$ & $64 / 122=52.5 \%$ \\
\hline \multicolumn{2}{c|}{ Recall } & $58 / 76=76.3 \%$ & $64 / 66=97.0 \%$ \\
\hline \hline
\end{tabular}

97 percent. Further analysis shows that, in basketball videos, other events, such as the trumpets of cheering squads or the audience and the grating between players' shoes and the floor, have similar behaviors as whistles because their energy concentrates on a small frequency region for a short time. The proposed whistle detection algorithm therefore also takes these events as whistle. Consequently, the precision of whistles becomes relatively poor, but we can still attain a very good recall value.

\subsection{Video Association Mining}

\subsubsection{The impact of TDT}

To figure out the relationship between $T D T$ and the features of mined associations, we set $T D T$ with different values $T(T=0,1,3,5,7,9$, and $\infty)$ and assess associations. For each mined association, we go through the video data to check whether each appearance of the association covers the happening of any event (ignoring the type of the event). We have defined four types of events for basketball videos, goal, foul, fast break, and successful defense. We define the EventCoverage $(E C)$ of the association as the ratio between the frequency of the association covering the happening of an event and the frequency of the association's appearances. The higher the $E C$, the better the association addresses the sports event information. Meanwhile, we have analyzed in Fig. 14 that the smaller the $T D T$, the less temporal support of mined associations because the appearances of many associations do not satisfy the constraint of $T D T$, so these associations are likely to be pruned out with the decrease of $T D T$. This indicates that TDT also acts as a factor for pruning associations and we need to evaluate the relationship between the percentage of the pruned association and the value of $T D T$ because, if $T D T$ is very sensitive to this percentage value (e.g., a small amount of decrease in $T D T$ results in a significant change with this percentage), we should provide a solution on how to select a suitable TDT value. For this purpose, we define the PruningRate $(P R)$ as the ratio between the numbers of associations when TDT is $T(T=0,1,3,5,7,9)$ and $\infty$, respectively. We run experiments on six basketball videos and present results in Fig. 18.

Fig. 18 demonstrates that, when the TDT increases, the associations likely become worse in addressing events. However, in the beginning part, the $E C$ increases with the increase of $T D T$. For example, when $T D T=1, E C$ has a larger value than when $T D T=0$. But, after that, $E C$ begins to drop. As we have introduced in Section 4.2, when $T D T=0$, the appearance of all associations should happen in one shot, which usually fails to mine associations a cross adjacent shots. For example, a goal event usually crosses two or three shots. One shot contains the goal action and applause, the following shot shows close-up frames of the

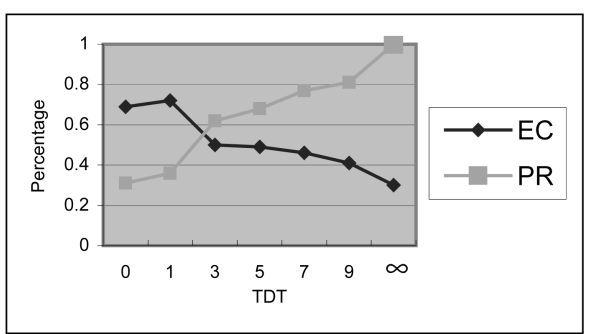

Fig. 18. The impact of $T D T$ values and association mining results.

shooter, and the scoreboard change may happen in the third shot. Hence, comparing $T D T=1$ and $T D T=0, E C$ has a little increase because more associations are mined out. However, comparing $T D T=1$ and $T D T=3$, the decline on $E C$ is dramatic. One possible reason is that sports events rarely cross more than three shots. When $T D T=3$ or larger, the appearances of some associations are actually useless in addressing sports events.

Fig. 18 also indicates that, in general, the smaller the $T D T$, the smaller is the number of mined associations. When comparing $T D T=1$ with $T D T=\infty$, about one third of associations are pruned out. It indicates that $T D T$ acts as an important factor in mining valuable video associations. A mining algorithm that ignores temporal information likely produces a large number of associations which cannot address video content and tend to be useless. A suitable $T D T$ is important in mining valuable associations from different types of video. We set $T D T=2$ in our system because our observation suggests that most sports events happen within three adjacent shots.

\subsubsection{Association-Based Events Detection}

To evaluate the performance of the association-based event detection scheme, we use four videos as the training set and two videos as the test set and present the average test results in Table 7. Here, $A N$ is the actual number of events contained in the video and $D N$ and $T N$ denote the numbers of events which have been detected and correctly detected, respectively. Precision and Recall are defined in (13):

$$
\text { Precision }=T N / D N ; \quad \text { Recall }=T N / A N .
$$

The results from Table 7 indicate that the proposed event detection strategy is promising in detecting three types of events (goal, foul, and fast break). Further analysis shows that these events have relatively distinct characteristics and can be characterized by various visual and audio cues. Among these three types of events, the results on "Foul" are relatively poor. Actually, we find

TABLE 7

Basketball Video Events Detection Results

\begin{tabular}{cccccc}
\hline \hline Events & $A N$ & $D N$ & $T N$ & Precision & Recall \\
\hline Goal & 42 & 49 & 38 & 0.776 & 0.905 \\
\hline Foul & 44 & 58 & 35 & 0.603 & 0.795 \\
\hline Fast Break & 29 & 35 & 24 & 0.686 & 0.827 \\
\hline Succ. Defense & 76 & 89 & 44 & 0.494 & 0.579 \\
\hline Average & 191 & 231 & 141 & 0.610 & 0.738 \\
\hline \hline
\end{tabular}


that almost all associations related to a foul contain referees' whistles and the camera motions, but such associations have been found to cover the events when a referee whistle is to resume the game. Hence, the precision of the foul is relatively poor in comparison with the goal and the fast break. Meanwhile, we find the results from the successful defense to be the worst among the four types of events. One reason is that this type of event is quite arbitrary and it's hard to characterize and distinguish it from other kinds of events, e.g., a steal or a miss. Meanwhile, the other three types of events have at least one unique tag that likely always associates with the happening of each event. The unique tags of a goal, foul, and fast break are a scoreboard change, a whistle of the referee, and a speeding up of the camera motion. However, for a successful defense, there is no such unique tag. Hence, the results of this type of event are the worst. But, the average results from Table 7 suggest that the proposed schemes are still promising to explore event information from sports videos.

\subsubsection{Association-Based Video Retrieval}

The results from the above sections have demonstrated the ability of video associations in covering video knowledge and our further analysis indicates that, due to the advantage of video associations in exploring the inherent correlations among video units, we can use associations to facilitate video retrieval. To demonstrate this fact, we design the following experiments:

Association-based database structures. For all videos in the database, we first adopt association mining to explore video associations (no association classification is involved at this stage). After that, we take the appearance of each association as one unit, and use the frames that correspond to the appearance of the association as the representative frames of the unit. So, each unit in the database is characterized by a set of representative frames $K_{1}, \ldots, K_{N}$. Instead of taking video shots as basic units of the retrieval, we use the frame regions corresponding to the video associations as the basic units.

Query generation and process. To generate query examples, we randomly select one video from the database and browse its content. Once we find an interesting continuous frame region, ${ }^{4}$ we mark this region and use it to retrieve from the database. To this end, we use a simple mechanism to select representative frames from the selected region: 1$)$ The first frame $\left(F_{1}\right)$ of the region is always taken as the first representative frame $R_{1}$ and we also take it as the reference frame $R F$ so far; 2) starting from the second frame, for each following frame $F_{l}$, we compare the difference between $F_{l}$ and $R F$ and, if their distance is larger than a threshold (which is controlled by users), we take $F_{l}$ as a representative frame $R_{k}$ and also take $F_{l}$ as the reference frame $R F$ so far; and (c), (a), and (b) are iteratively executed until all frames in the query region are processed. When comparing users' query (which consists of a set of representative frames $R_{1}, . . R_{M}$ ) with a unit in the database (each unit is denoted by a set of representative frames $\left.K_{1}, \ldots, K_{N}\right)$, we adopt the similarity function defined by (14), where $\operatorname{Dis}()$ is defined by (1). Actually, (14) is the average similarity of representative frames in the query region and

4. Most likely, the selected region should contain an interesting event, which implies users' query interests. In our experiments, the selected region contains one of the four events in Table 6, but this query region does not necessarily cover the whole event.

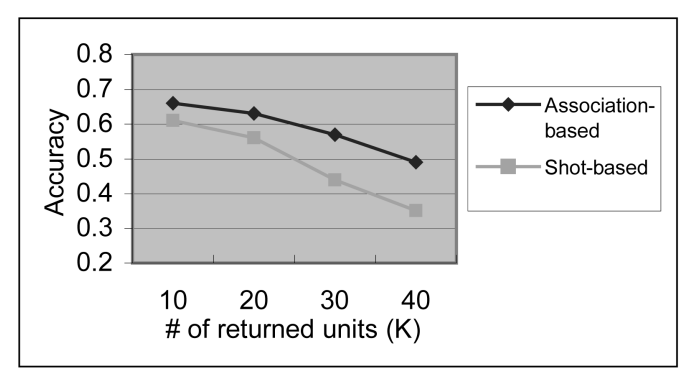

Fig. 19. Average retrieval accuracy comparison.

their most similar representative frames of the unit in the database:

$$
\text { QuerySim }=1-\frac{\sum_{l=1}^{M} \operatorname{Min}\left\{\operatorname{Dis}\left(R_{l}, K_{j}\right), j=1, \ldots, N\right\}}{M} \text {. }
$$

System performance measures. For comparison, we also perform retrieval on a shot-based database, where basic units of the retrieval are video shots and one key-frame (the 10th frame) is selected to characterize the content of each shot. Each time the user triggers a query, we use the same query region to retrieve from the database by using the above two mechanisms, respectively. Note that, with the shot-based approach, the retrieved units are video shots, but, with the association-based scheme, the results are frame regions corresponding to associations. We evaluate the retrieval accuracy, (15), in the top- $K$ results, where a "relevant unit" means a unit (or shot) with similar content as the query region:

$$
\text { Accuracy }=\frac{\text { \# of relevant units in top } K \text { returns }}{K} \text {. }
$$

With (15), the unit which is longer in length has a higher possibility of being "relevant" to the query. But, this is actually less attractive from the users' point of views because users need to explore the results to find the part they are actually interested in. An intuitive sense is that users prefer the units with a short length but which are still relevant to the query. So, we provide the following two measures, Average Unit length Ratio (AUR) and Average Relevant unit length Ratio $(A R R)$, which are defined by (16):

$$
\begin{aligned}
& A U R=\frac{\text { Avg \# of frames in top } K \text { returns }}{\# \text { of frames in the query region }} \\
& A R R=\frac{\text { Avg \# of frames in relevant return units }}{\# \text { of frames in the query region }} .
\end{aligned}
$$

System performance analysis. We perform retrieval on eight sports videos where query regions are taken from the videos which are already in the database. We execute queries for about 80 times and, for each query, we will adjust $K$ to get different results, and report the results in Fig. 19 and Table 8.

As we can see from Fig. 19, when using video associations to construct the database, the retrieval results are significantly better than the shot-based approach. Take $K=20$ as an example (the system allows a return of 20 results). The result from the association-based approach is 8 percent better than the shot-based database. This improvement becomes more significant when the values of $K$ increase. This indicates that, by integrating video 
TABLE 8

Average Length Ratios between Results and Query

\begin{tabular}{cc|c|c|c|c}
\hline \hline \multirow{2}{*}{ AUR } & & $K=10$ & $K=20$ & $K=30$ & $K=40$ \\
\cline { 2 - 6 } & Association & 2.12 & 2.08 & 1.93 & 1.62 \\
\hline \multirow{2}{*}{ ARR } & Shot & 14.66 & 13.47 & 12.89 & 11.04 \\
\cline { 2 - 6 } & Association & 1.87 & 1.61 & 1.56 & 1.78 \\
\cline { 2 - 6 } & Shot & 16.88 & 15.26 & 14.97 & 12.14 \\
\hline \hline
\end{tabular}

associations, we can actually improve video retrieval systems by providing users with the results which are semantically related to their queries. Despite the improvement from the association-based mechanism, we are actually surprised that the results from the shot-based approach are much better than what we thought. For example, when $K=10$, about 60 percent of the retrieved shots are actually relevant to users' queries. Nevertheless, further analysis indicates that, for sports videos, each "court field shot" is actually quite long, which likely covers user's query by chance. The results from Table 8 indicate this fact. As we can see, on average, with the shot-based approach, the returned units (video shots) are over 10 times longer than users' query. It indicates that users will have to explore the retrieval results to find the part they are actually interested in (and, because the result shots are long, they have a high possibility of matching users' query). With the association-based mechanism, the retrieved units are likely close to the length of the query, so the users may "directly" find out the content they want. We noticed that, when increasing the value $K$, both $A U R$ and $A R R$ from two approaches tend to decrease. One possible reason is that, with the increase of $K$, the average length of the retrieved units is actually closer to the average length of all units in the database and lots of short units (or shots) in the video will have an impact to the average length of the retrieval results.

\subsection{Knowledge-Based Video Indexing}

Once video association mining and classification have been finalized, we can directly use associations to support category-based hierarchical video browsing and knowledge-based video indexing for efficient video retrieval and content management. In this section, we first analyze the complexity of the proposed indexing framework, and then evaluate its performance on real-world data.

\subsubsection{Complexity Analysis}

With the general knowledge-based indexing framework demonstrated in Fig. 1, the search time $T_{e}$ for retrieving video from a large-scale database is the sum of two components: 1) time $T_{s}$ for comparing the relevant video shots in the database and 2) time $T_{r}$ for ranking the relevant results. If no database indexing structure is used for organizing this search procedure, the total retrieval time is:

$$
T_{e}=T_{s}+T_{r}=N_{T} \cdot T_{m}+O\left(N_{T} \log N_{T}\right),
$$

where $N_{T}$ is the number of videos in the database, $T_{m}$ is the basic time to calculate the $m$-dimensional feature-based distance between two video shots, and $O\left(N_{T} \log N_{T}\right)$ is the time to rank $N_{T}$ elements.

Our knowledge-based multilevel video indexing structure can provide fast retrieval because only the relevant database management units are compared with the query example. Moreover, the dimension reduction techniques can be utilized to guarantee that only the discriminating features are selected for video representation and indexing and, thus, the basic time for calculating the feature-based similarity distance is also reduced. With the proposed video indexing structure, assume $M_{c}, M_{s c}$, and $M_{s}$ are the numbers of the nodes at the cluster level, the most relevant subcluster, and the scene levels, respectively, $M_{o}$ is the number of video shots that reside in the most relevant scene node. Assume further that $T_{c}, T_{s c}, T_{s}$, and $T_{o}$ are the basic times for calculating the similarity distances in the corresponding feature subspace. (It is obvious that $T_{c}, T_{s c}$, $T_{s}, T_{o} \leq T_{m}$ because only the discriminating features are used.) Then, the total retrieval time for our cluster-based indexing system $T_{e}^{\prime}$ is given by (18):

$$
T_{e}^{\prime}=M_{c} \cdot T_{c}+M_{s c} \cdot T_{s c}+M_{s} \cdot T_{s}+M_{o} \cdot T_{o}+O\left(M_{o} \log M_{o}\right),
$$

where $O\left(M_{o} \log M_{o}\right)$ is the total time for ranking the relevant shots residing in the corresponding scene node. Since $\left(M_{c}+M_{s c}+M_{s}+M_{o}\right) \ll N_{T}, \quad\left(T_{c}, T_{s c}, T_{s}, T_{o}\right) \leq T_{m}$, thus $T_{e}^{\prime} \ll T_{e}$.

\subsubsection{Knowledge-Based Indexing Results}

To demonstrate the performance of the proposed knowledge-based video indexing framework, we compare the CPU cost of video retrieval between the proposed indexing framework and exhaustive search. We did not compare our work with other indexing techniques such as R-tree [42], SR-tree [43] etc., because all these indexing techniques are efficient when the feature dimension is relatively low. As suggested by Cui et al. [41], when the number of dimensions is over 30 , the exhaustive search turns to be the best, given that the data in the data set is uniformly distributed. In our case, each key-frame is represented by a 266-dimensional vector and this number is much higher than 30 .

Database indexing structures. When evaluating the performance of the proposed indexing structure, we repeat the experiment in Section 6.2.3 (association-based video retrieval) with the following modification: After association mining from all videos, we adopt association classification to classify each association (note that Section 6.2.3 does not conduct this step). Then, we use the semantic category of the identified associations and the index structure in Fig. 2 to construct our database.

Query generation and process. When users specify a query frame region from a video which does not exist in the database, we first preprocess the video to mine and classify associations and then use the associations in the region to find video units in the database which have the same category as the query and, after that, we use (14) to find the most similar units and return the results (if the query video already exists in the database, we can directly use the video associations, which have been mined before, to facilitate the query). In the case where the user-specified region does not contain any association, we will use the same approach in Section 6.2.3 to retrieve from the database (i.e., we ignore the index structure and execute an exhaustive search).

System performance measures. For comparative studies, we implement and compare with the exhaustive search mechanism, i.e., exactly the same approach in Section 6.2.3. Whenever users trigger a query, the same query region is 


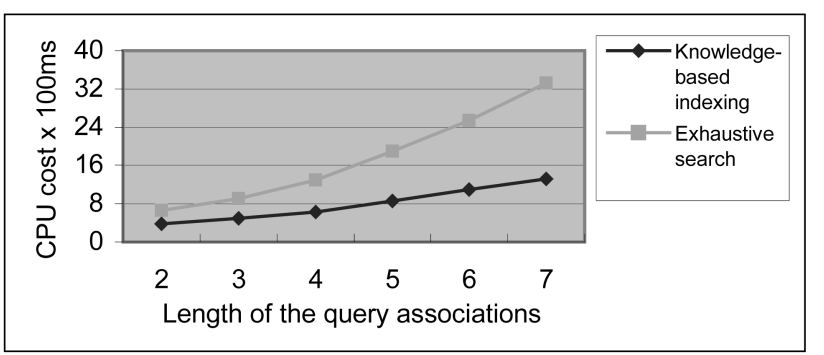

Fig. 20. CPU cost comparisons between knowledge-based indexing and exhaustive search.

used for two approaches to retrieve from the database. We use average CPU time expense to evaluate two methods, where, for knowledge-based indexing, the CPU time includes the time to mine and classify associations (this one time overhead expense is paid off the first time we select the query from a new video).

System performance analysis. Obviously, the actual CPU expense is determined by four most important factors:

1. the number of the levels of the index structure in Fig. 2,

2. the size of the feature subset in evaluating the distance,

3. the size of the semantic concept at each index level, and

4. the complexity of the query (the longer the query association, the higher is the CPU cost).

We execute retrieval about 300 times in total and evaluate the average CPU cost. For each query, we count the association length in the query region and the corresponding query time, and provide results in Fig. 20, where the $y$ axis represents the CPU cost and the $x$-axis denotes the length of the query associations.

The experimental results in Fig. 20 demonstrate that, with the increase of the length of the query associations, both mechanisms suffer from the increase of the CPU expense because a longer length of associations implies more complex query patterns and more time is needed for response. Although exhaustive search does not use associations, the longer the length of the query associations, the more complicated the content of the query region and the more representative frames will be detected for exhaustive search and, accordingly, is more time for retrieval involved. As shown in Fig. 20, with the proposed knowledge-based indexing framework, we can significantly improve the efficiency of a video retrieval system, where the more complicated the query, the more improvement can be achieved. As we can see, when the length of the association in the user's query is 7 , the proposed index framework is about 3 times better than exhaustive search. On average, the system improvement is about 2.2 times better in comparison with exhaustive search. Currently, we have only implemented two levels and four concept categories in the index structure and we believe that, by integrating more levels and concepts, the contribution of the proposed index framework can be more significant.

\section{Conclusions and Remarks}

In this paper, we have proposed a solution for a new research area of video mining-video association mining.
We have used video associations to construct a knowledgebased video indexing structure to support efficient video database management and access. We have introduced various techniques to extract visual and audio semantic cues and combined them into one hybrid stream by considering their original temporal order in the video. Consequently, the video data is transformed into a relational data set. We have employed a sequential multi level association mining strategy to mine associated video items and take them as video associations. We have adopted a scheme to classify associations into different categories, where each association can possibly indicate the happening of one type of event. The knowledge-based video indexing structure is accomplished by mining and classifying associations from video data. We have presented experimental results to demonstrate the performance of the proposed schemes. We believe we have explored a new research area to discover video knowledge for efficient video database management.

While the strategies presented in this paper are specific to basketball videos, mining associations for video knowledge exploration is an essential idea we want to convey here. From this point of view, further research could be conducted on the following aspects: 1) Extend the current framework to other domains and evaluate the performance of the video mining algorithm in environments with more events. We believe the most promising domain is the surveillance video, where the routine vehicles in security areas normally comply with some associations like enter $\rightarrow$ stop $\rightarrow$ drop off $\rightarrow$ leave and a vehicle which does not comply with this association might be problematic and deserves further investigation. However, due to the inherent differences between different video domains (e.g., the concept of shot and video text do not exist in surveillance videos), we may need more activities to analyze the video content details for association mining, e.g., extract trails and status of moving objects to characterize associations. 2) We have adopted various video processing techniques to explore visual and audio cues for association mining and it will inevitably incur information loss from the original video sequences to transferred symbolic streams; more studies are needed to address this issue in the mining activities. 3) The mining algorithms in this paper are mainly derived from the existing data mining schemes (with some extensions for video mining scenarios); extensive studies are needed to explore efficient mining algorithms which are unique for mining knowledge from video data.

\section{ACKNOWLEDGMENT}

The authors would like to thank the anonymous reviewers for their constructive comments on two earlier versions of this paper. This work was supported by the US Army Research Laboratory and the US Army Research Office under grant number DAAD19-02-1-0178, the US National Science Foundation under grants 0093116-IIS and 9972883EIA, and the NSF under contract 69935010.

\section{REFERENCES}

[1] H. Zhang, A. Kantankanhalli, and S. Smoliar, "Automatic Partitioning of Full-Motion Video," ACM Multimedia Systems, vol. 1, no. 1, pp. 10-28, 1993.

[2] A. Yoshitaka and T. Ichikawa, "A Survey on Content-Based Retrieval for Multimedia Databases," IEEE Trans. Knowledge and Data Eng., vol. 11, no. 1, pp. 81-93, Jan./Feb. 1999. 
[3] H. Jiang and A.K. Elmagarmid, "WVTDB-A Semantic ContentBased Video Database System on the World Wide Web," IEEE Trans. Knowledge and Data Eng., vol. 10, no. 6, pp. 947-966, Nov./ Dec. 1998.

[4] C. Snoek and M. Worring, "Multimodal Video Indexing: A Review of the State-of-the-Art," Multimedia Tools and Applications, to be published in 2005.

[5] F. Kokkoras, H. Jiang, I. Vlahavas, A. Elmagarmid, E. Houstis, and W. Aref, "Smart VideoText: A Video Data Model Based on Conceptual Graphs," ACM/Springer Multimedia Systems, vol. 8, no. 4, pp. 328-338, 2002.

[6] X. Zhu, J. Fan, W.G. Aref, and A.K. Elmagarmid, "ClassMiner: Mining Medical Video Content Structure and Events Towards Efficient Access and Scalable Skimming," Proc. ACM SIGMOD Workshop, pp. 9-16, 2002.

[7] X. Zhu, W. Aref, J. Fan, A. Catlin, and A. Elmagarmid, "Medical Video Mining for Efficient Database Indexing, Management and Access," Proc. 19th Int'l Conf. Data Eng., pp. 569-580, 2003.

[8] Y. Matsuo, K. Shirahama, and K. Uehara, "Video Data Mining. Extracting Cinematic Rules from Movie," Proc. Int'l Workshop Multimedia Data Management (MDM-KDD), 2003.

[9] R.R. Wang and T.S. Huang, "A Framework of Human Motion Tracking and Event Detection for Video Indexing and Mining," Proc. DIMACS Workshop Video Mining, 2002.

[10] J. Oh and B. Bandi, "Multimedia Data Mining Framework for Raw Video Sequence," Proc. Int'l Workshop Multimedia Data Management (MDM-KDD), 2002.

[11] J. Pan and C. Faloutsos, "VideoCube: A Novel Tool for Video Mining and Classification," Proc. Int'l Conf. Asian Digital Libraries (ICADL), pp. 194-205, 2002

[12] J. Pan and C. Faloutsos, "GeoPlot: Spatial Data Mining on Video Libraries," Proc. Int'l Conf. Information and Knowledge Management, pp. 405-412, 2002.

[13] X. Zhu and X. Wu, "Mining Video Association for Efficient Database Management," Proc. Int'l Joint Conf. Artificial Intelligence, pp. 1422-1424, 2003.

[14] X. Zhu and X. Wu, "Sequential Association Mining for Video Summarization," Proc. IEEE Int'l Conf. Multimedia and Expo, vol. 3, pp. 333-336, 2003.

[15] J. Fan, X. Zhu, and X. Lin, "Mining of Video Database," Multimedia Data Mining, 2002.

[16] L. Xie, S.-F. Chang, A. Divakaran, and H. Sun, "Unsupervised Mining of Statistical Temporal Structures in Video," Video Mining, A. Rosenfeld, D. Doremann, and D. Dementhon eds., Kluwer Academic, 2003.

[17] D. Wijesekera and D. Barbara, "Mining Cinematic Knowledge: Work in Progress," Proc. Int'l Workshop Multimedia Data Management (MDM-KDD), 2000.

[18] M. Windhouwer, R. Zwol, H. Blok, W. Jonker, M. Kersten, and P. Apers, "Content-Based Video Indexing for the Support of Digital Library Search," Proc. Int'l Conf. Data Eng., pp. 494-495, 2002.

[19] S. Newsam, J. Tesic, L. Wang, and B.S. Manjunath, "Mining Images and Video," Proc. DIMACS Workshop Video Mining, 2002.

[20] R. Agrawal and R. Srikant, "Fast Algorithms for Mining Association Rules," Proc. Very Large Data Bases Conf., pp. 487499, 1994

[21] R. Agrawal and R. Srikant, "Mining Sequential Patterns," Proc. 11th Int'l Conf. Data Eng., 1995.

[22] J. Han and M. Kamber, Data Mining: Concepts and Techniques. Morgan Kaufmann, 2000.

[23] B. Thuraisingham, Managing and Mining Multimedia Database. CRC Press, 2001.

[24] O. Zaiane, J. Han, Z. Li, S. Chee, and J. Chiang, "MultimediaMiner: A System Prototype for Multimedia Data Mining," Proc. ACM SIGMOD, pp. 581-583, 1998.

[25] S. Nepal, U. Srinivasan, and G. Reynolds, "Automatic Detection of 'Goal' Segments in Basketball Videos," Proc. Ninth ACM Multimedia Conf., pp. 261-269, 2001.

[26] L. Duan, M. Xu, T. Chua, Q. Tian, and C. Xu, "A Mid-Level Representation Framework for Semantic Sports Video Analysis," Proc. of 11th ACM Multimedia Conf., pp. 33-44, 2003.

[27] J. Fan, W.G. Aref, A.K. Elmagarmid, M. Hacid, M. Marzouk, and X. Zhu, "MultiView: Multi-Level Video Content Representation and Retrieval," J. Electronic Imaging, vol. 10, no. 4, pp. 895-908, 2001
[28] W. Zhou, A. Vellaikal, and C. Kuo, "Rule-Based Video Classification System for Basketball Video Indexing," Proc. ACM Multimedia Workshops, pp. 213-216, 2000.

[29] L. Xie, S. Chang, A. Divakaran, and H. Sun, "Structure Analysis of Soccer Video with Hidden Markov Models," Proc. IEEE Int'l Conf. Acoustics, Speech, and Signal Processing (ICASSP), 2002.

[30] W. Wolf, "Key Frame Selection by Motion Analysis," Proc. IEEE Int'l Conf. Acoustics, Speech, and Signal Processing (ICASSP), pp. 1228-1231, 1996.

[31] H. Tamura, S. Mori, and T. Yamawaki, "Texture Features Corresponding to Visual Perception," IEEE Trans. Systems, Man, and Cybernetics, vol. 8, no. 6, pp. 460-473, 1978

[32] S. Horowitz and T. Pavlidis, "Picture Segmentation by a Directed Split-and-Merge Procedure," Proc. Int'l Joint Conf. Pattern Recognition, pp. 424-433, 1974

[33] WOCAR Engine 2.5, http://ccambien.free.fr/wocar/, 2004.

[34] A. Jain and B. Yu, "Automatic Text Location in Images and Video Frames," Pattern Recognition, vol. 31, no. 12, pp. 2055-2076, 1998.

[35] X. Zhu, A.K. Elmagarmid, X. Xue, L. Wu, and A. Catlin, "InsightVide: Towards Hierarchical Video Content Organization for Efficient Browsing, Summarization, and Retrieval," IEEE Trans. Multimedia, 2004.

[36] T. Oates and P. Cohen, "Searching for Structure in Multiple Streams of Data," Proc. 13th Int'l Conf. Machine Learning, pp. 346354,1996

[37] J. Han, G. Dong, and Y. Yin, "Efficient Mining Partial Periodic Patterns in Time Series Database," Proc. Int'l Conf. Data Eng., pp. 106-115, 1999.

[38] R. Srikant and R. Agrawal, "Mining Generalized Association Rules," Proc. 21th Very Large Data Bases Conf., 1995.

[39] R. Gwadera, M. Atallah, and W. Szpankowski, "Reliable Detection of Episodes in Event Sequences," Proc. Third Int'l Conf. Dato Mining, pp. 67-74, 2003.

[40] T. Cormen, C. Leiserson, R. Rivest, and C. Stein, Introduction to Algorithms. MIT Press, 2001.

[41] B. Cui, B. Ooi, J. Su, and K. Tan, "Contorting High Dimensional Data for Efficient Main Memory Processing," Proc. SIGMOD Conf., pp. 479-490, 2003.

[42] A. Guttman, "R-Trees: A Dynamic Index Structure for Spatial Searching," Proc. SIGMOD Conf., pp. 47-57, 1984.

[43] N. Katayama and S. Satoh, "The SR-Tree: An Index Structure for High-Dimensional Nearest Neighbor Queries," Proc. SIGMOD Conf., pp. 369-380, 1997.

[44] W. Hsu, J. Dai, and M. Lee, "Mining Viewpoint Patterns in Image Databases," Proc. SIGKDD, pp. 553-558, 2003.

[45] H. Mannila, H. Toivonen, and A. Verkamo, "Discovery of Frequent Episodes in Event Sequences," Data Mining and Knowledge Discovery, vol. 1, no. 3, pp. 259-289, 1997.

[46] R. Srikant and R. Agrawal, "Mining Sequential Patterns: Generalizations and Performance Improvements," Proc. Fifth Int'l Conf. Extending Database Technology (EDBT), 1996.

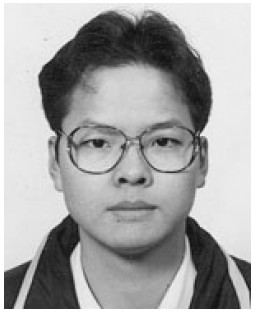

Xingquan Zhu received the $\mathrm{PhD}$ degree in computer science from Fudan University, Shanghai, China, in 2001. He is a research assistant professor in the Department of Com puter Science at the University of Vermont. He spent four months with Microsoft Research Asia Beijing, China, where he was working on content-based image retrieval with relevance feedback. From 2001 to 2002, he was a postdoctoral associate in the Department of Computer Science, Purdue University, West Lafayette, Indiana. His research interests include data mining, machine learning, data quality, multimedia systems, and information retrieval. Since 2000, Dr. Zhu has published extensively, including more than 40 refereed papers in various journals and conference proceedings. $\mathrm{He}$ is a member of the IEEE. 


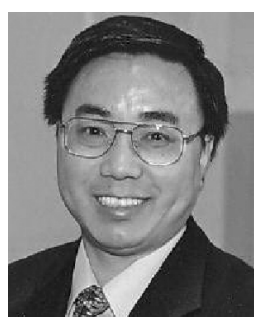

Xindong $\mathbf{W u}$ received the $\mathrm{PhD}$ degree in artificial intelligence from the University of Edinburgh, Britain. He is a professor and chair of the Department of Computer Science at the University of Vermont. His research interests include data mining, knowledge-based systems, and Web information exploration. He has published extensively in these areas in various journals and conferences. He has been an invited/keynote speaker at six international conferences. Dr. Wu is the Editor-in-Chief of the IEEE Transactions on Knowledge and Data Engineering, executive editor (1 January 1999-31 December 2004) and an honorary Editor-in-Chief (starting 1 January 2005) of Knowledge and Information Systems (a peer-reviewed archival journal published by Springer-Verlag), the founder and current steering committee chair of the IEEE International Conference on Data Mining, a series editor of the Springer Book Series on Advanced Information and Knowledge Processing (AI\&KP), and the chair of the IEEE Computer Society Technical Committee on Computational Intelligence (TCCI). He is the winner of the 2004 ACM SIGKDD Service. He is a senior member of the IEEE.

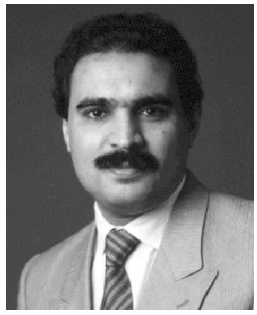

Ahmed K. Elmagarmid received the BS degree in computer science from the University of Dayton and the MS and PhD degrees from The Ohio State University in 1977, 1981, and 1985, respectively. He received a Presidential Young Investigator award from the US National Science Foundation and the distinguished alumni awards from Ohio State University and the University of Dayton in 1988, 1993, and 1995, respectively. Professor Elmagarmid is the editorin-chief of Distributed and Parallel Databases: An International Journal and of the book series on Advances in Database Systems. He serves on the editorial boards of: Information Sciences and Journal of Communications Systems. He has served on the editorial boards of the IEEE Transactions on Knowledge and Data Engineering, IEEE Transactions on Computers, and the IEEE Data Engineering Bulletin. His research interests are in the areas of video databases, multidatabases, data quality, and their applications in telemedicine and digital government. $\mathrm{He}$ is the author of several books in databases and multimedia. He was chief scientist for Hewlett-Packard from 2001 to 2003 while on leave from Purdue. He has served widely as an industry consultant and/or adviser to Telcordia, Harris, IBM, MCC, UniSql, MDL, BNR, etc. He has served as a faculty member at the Pennsylvania State University from 1985-1988 and has been with the Department of Computer Science at Purdue University since 1988. He is a senior member of the IEEE.

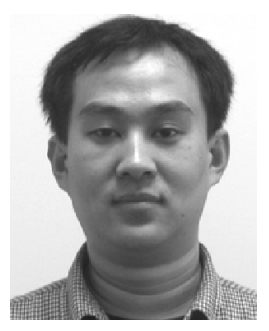

Zhe Feng received the $\mathrm{PhD}$ degree in computer science from Fudan University, Shanghai, China, in 2004. He is now a research assistant in the Department of Computer Science, Fudan University. His research interests include audio/ video content analysis and multimedia information retrieval.

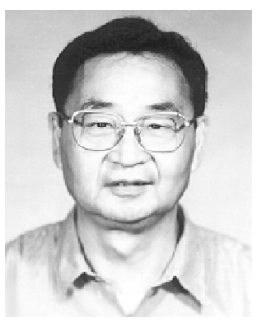

Lide Wu graduated from the Department of Mathematics at Fudan University, and was working there until 1975. Since then, he has been with the Department of Computer Science at the same university and is now a chair professor there. He was the chairman of the department during 1982-1985. His research interests include image processing, video processing, computer vision, pattern recognition, and Chinese text processing. He is the author or coauthor of more than 10 books and more than 200 papers. He is vice chairman of technical committee of Al \& PR of Chinese Computer Society, a senior member of the IEEE, and a member of the ACM and the New York Academy of Sciences.

For more information on this or any other computing topic, please visit our Digital Library at www.computer.org/publications/dlib. 